\title{
Ionospheric conductances derived from satellite measurements of auroral UV and X-ray emissions, and ground-based electromagnetic data: a comparison
}

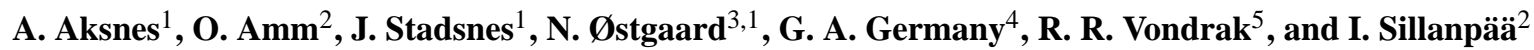 \\ ${ }^{1}$ Department of Physics and Technology, University of Bergen, Bergen, Norway \\ ${ }^{2}$ Finnish Meteorological Institute, Geophysical Research Division, P.O. Box 503, FIN-00101 Helsinki, Finland \\ ${ }^{3}$ University of California, Berkeley, CA 94720-7450, USA \\ ${ }^{4}$ University of Alabama in Huntsville, AL 35899, USA \\ ${ }^{5}$ NASA/Goddard Space Flight Center, Greenbelt, MD 20771, USA
}

Received: 17 January 2004 - Revised: 5 October 2004 - Accepted: 19 October 2004 - Published: 28 February 2005

\begin{abstract}
Global instantaneous conductance maps can be derived from remote sensing of UV and X-ray emissions by the UVI and PIXIE cameras on board the Polar satellite. Another technique called the 1-D method of characteristics provides mesoscale instantaneous conductance profiles from the MIRACLE ground-based network in Northern Scandinavia, using electric field measurements from the STARE coherent scatter radar and ground magnetometer data from the IMAGE network. The method based on UVI and PIXIE data gives conductance maps with a resolution of $\sim 800 \mathrm{~km}$ in space and $\sim 4.5 \mathrm{~min}$ in time, while the 1-D method of characteristics establishes conductances every $20 \mathrm{~s}$ and with a spatial resolution of $\sim 50 \mathrm{~km}$. In this study, we examine three periods with substorm activity in 1998 to investigate whether the two techniques converge when the results from the 1-D method of characteristics are averaged over the spatial and temporal resolution of the UVI/PIXIE data.
\end{abstract}

In general, we find that the calculated conductance sets do not correlate. However, a fairly good agreement may be reached when the ionosphere is in a state that does not exhibit strong local turbulence. By defining a certain tolerance level of turbulence, we show that 14 of the 15 calculated conductance pairs during relatively uniform ionospheric conditions differ less than $\pm 30 \%$. The same is true for only 4 of the 9 data points derived when the ionosphere is in a highly turbulent state. A correlation coefficient between the two conductance sets of 0.27 is derived when all the measurements are included. By removing the data points from time periods when too much ionospheric turbulence occurs, the correlation coefficient raises to 0.57 . Considering the two very different techniques used in this study to derive the conductances, with different assumptions, limitations and scale sizes, our results indicate that simple averaging of mesoscale results allows a continuous transition to large-scale results.

Correspondence to: A. Aksnes

(arve.aksnes@ift.uib.no)
Therefore, it is possible to use a combined approach to study ionospheric events with satellite optical and ground-based electrodynamic data of different spatial and temporal resolutions. We must be careful, though, when using these two techniques during disturbed conditions. The two methods will only give results that systematically converge when relatively uniform conditions exist.

Key words. Ionosphere (Auroral ionosphere; Particle precipitation; Instruments and techniques)

\section{Introduction}

Knowledge of the ionospheric conductivity is needed to understand the ionospheric electrodynamics and the dynamical magnetosphere-ionosphere (MI) coupling. The heightintegrated Hall and Pedersen conductivities can be strongly enhanced during auroral substorms, when the particle precipitation increases the electron density in the $E$-layer. The ionospheric conductivity pattern is complicated, though, as the precipitation may vary strongly in time and space.

The Hall and Pedersen conductivities, $\sigma_{H}$ and $\sigma_{P}$, can be derived using the classical expressions (Krall and Trivelpiece, 1973):

$\begin{aligned} \sigma_{H} & =\frac{N_{e} e}{B}\left(\frac{\Omega_{e}^{2}}{v_{e n \perp}^{2}+\Omega_{e}^{2}}-\frac{\Omega_{i}^{2}}{v_{i n}^{2}+\Omega_{i}^{2}}\right) \\ \sigma_{P} & =\frac{N_{e} e}{B}\left(\frac{v_{i n} \Omega_{i}}{v_{i n}^{2}+\Omega_{i}^{2}}+\frac{v_{e n \perp} \Omega_{e}^{2}}{v_{e n \perp}^{2}+\Omega_{e}^{2}}\right),\end{aligned}$

where $e$ denotes the elementary charge, $v_{\mathrm{en} \perp}$ represents the collision frequency between electrons and neutrals for motions perpendicular to the magnetic field $\boldsymbol{B}, v_{i n}$ is the (isotropic) collision frequency between ions and neutrals, and $\Omega_{e}\left(\Omega_{i}\right)$ is the gyrofrequency for electrons (ions). 
Note that Eqs. (1) and (2) are applied to a pseudo-ion average (Brekke, 1997), derived by proper weighting of all ions. The most critical parameter, though, is the electron density $N_{e}$. During an auroral substorm, $N_{e}$ may vary strongly, resulting in large spatial and temporal changes in the conductivities. $N_{e}$ is related to the particle precipitation, and height profiles of the ionization can be estimated using measurements from particle detectors on rockets (Marklund et al., 1982; Opgenoorth et al., 1983) and satellites (Vondrak and Robinson, 1985). This procedure involves a theoretical modelling of the interaction of the precipitating particles with atmospheric constituents. Another approach is to calculate the conductivities from measurements of $N_{e}$ by incoherent scatter radars on the ground. Numerous studies in the literature have performed such an investigation, including incoherent scatter radar measurements from Chatanika (Brekke et al., 1974; Banks and Doupnik, 1975; Wedde et al., 1977; de la Beaujardière et al., 1981; Kamide and Vickrey, 1983; Robinson and Vondrak, 1984), Sondrestrom (Robinson et al., 1987; Watermann et al., 1993;), and EISCAT (Buchert et al., 1988; Schlegel, 1988; Brekke et al., 1989; Senior, 1991; Olsson et al., 1996; Lühr et al., 1998; Davies and Lester, 1999).

An advantage with both in-situ particle measurements on rockets or satellites, and $N_{e}$ calculated from incoherent scatter radars, is the high resolution in time and space. The disadvantage, though, is that only local measurements can be obtained. To provide a global map of conductances, remote sensing of visible, ultraviolet and X-ray emissions produced by the precipitating particles has been shown to be a powerful tool.

Kamide et al. (1986) used images of auroral emissions observed with the Dynamic Explorer (DE)-1 satellite to calculate ionospheric height-integrated conductivities, or conductances, over the polar region with a 12-min time resolution. A direct empirical relationship between the conductances and the auroral ultraviolet (UV) emission intensity was assumed, in order to perform the calculations. Lummerzheim et al. (1991) also used auroral images from the DE-1 satellite to produce global conductance patterns. Their technique to calculate the height-integrated conductivities was somewhat different, as they first derived the characteristic energy and flux of the incoming particles from UV (OI lines at 130.4 and $135.6 \mathrm{~nm})$ and visible $(557.7 \mathrm{~nm})$ emissions. Then a method developed by Rees et al. (1988) was used in order to convert the energy spectra to conductance values.

The precipitating electron energy determines the height region of energy deposition, being at lower altitudes with higher energies (Rees, 1963). The intensity of the shortest UV-emission wavelengths escaping the atmosphere decreases strongly due to absorption of $\mathrm{O}_{2}$, when produced by energetic electrons depositing their energy too low in the atmosphere (Lummerzheim et al., 1991; Germany et al., 1990; 1998b). This means that measurements of UV-emissions are unable to accurately characterize the most energetic electrons of more than $\sim 10 \mathrm{keV}$ (Robinson and Vondrak, 1994). Østgaard et al. (2001; 2002) combined UV-emissions from the Ultraviolet Imager (UVI) and X-ray data from the Polar
Ionospheric X-ray Imaging Experiment (PIXIE) on board the Polar satellite, to derive global maps of the precipitating electron energy spectra in an energy range of $\sim 0.1-100 \mathrm{keV}$. By using a computer code based on the TANGLE code (Vondrak and Baron, 1976; Vondrak and Robinson, 1985), Aksnes et al. $(2002 ; 2004)$ used this data base to derive height profiles of the ionization and the height-integrated conductivities by applying the empirical formulas given in Eqs. (1) and (2). It was found that UV measurements are sufficient to characterize the lower electron energies contributing to the Pedersen conductance, while X-ray measurements are needed to characterize the energetic electrons affecting the Hall conductance.

Another approach to derive the ionospheric conductances is to make use of simultaneous ground magnetic and ionospheric electric field data. The disturbances of the magnetic field $\boldsymbol{B}$ measured on the ground depends on the horizontal electric current density $\boldsymbol{J}$ flowing in the ionosphere, which is related to the electric field $\boldsymbol{E}$ through Ohm's law involving the Hall and Pedersen conductances.

Applying a "trial and error scheme" (e.g. Baumjohann et al., 1981; Opgenoorth et al., 1983) different values of the conductances are combined with models of the ground magnetic and ionospheric electric field until the calculated and measured magnetic disturbances converge to a sufficient degree. The method is applicable even during periods with very poor data coverage, but the method does not give unique solutions and no error estimates can be calculated. Another problem with the "trial and error" approach is that the electrodynamical parameters must be modelled on a much larger area than the region of interest to reproduce magnetic disturbances. This often requires that one has to assume a stationary structure moving with a certain velocity over the field of view of the measurements.

Another technique to investigate the electrodynamics is the method of characteristics (Inhester et al., 1992; Amm, 1995; 1998; Sillanpää, 2002). This is a forward modelling method which gives more precise results than the "trial and error" approach (Untiedt and Baumjohann, 1993). Using measurements of the ground magnetic and ionospheric electric field to solve a first-order differential equation, the Hall conductance is derived. By then assuming a ratio between the Hall and Pedersen conductances, the remaining electrodynamical quantities are inferred. There are regions where the solutions using the method of characteristics are not unique. Such regions, though, are known and estimates of the errors can be made. Another advantage compared with the "trial and error" approach is that no data are needed outside the region of interest.

In order to understand the coupling between the ionosphere and the magnetosphere, it would be of great value to combine ground-based measurements with observations from instruments on satellites in space. The many various instruments and techniques differ in applicability, resolution schemes and reliability. The latter indicates that comparison studies are sometimes needed to verify the calculation of different electrodynamical quantities. A number of com- 
parison studies exist in the literature (e.g. Basu and Jasperse, 1987; Vondrak et al., 1988; Senior, 1991; Watermann et al., 1993; Doe et al., 1997; Germany et al., 1997; Dymond et al., 2001; Østgaard et al., 2001). Such studies are complicated when the stochastical processes operate on different scales. Given two methods that measure a parameter with different spatial resolution, it is not a priori clear that an averaging of the results of the method with finer resolution reproduces the ones of the method with coarser resolution. This possible scale-dependency has not been given much attention, though, in earlier papers. For a detailed mathematic formulation in terms of properties of statistical processes, the reader is referred to work by Christakos (1992).

In this paper, we derive and compare the ionospheric Hall and Pedersen conductances above Northern Scandinavia using two different techniques. The first method is the procedure by Aksnes et al. (2002; 2004), based on remote sensing of UV and X-ray emissions from space, as well as the classical expressions of the conductivities given in Eq. (1) and Eq. (2). This technique will be described more thoroughly in Sect. 2.1. The other procedure is the method of characteristics (Inhester et al., 1992; Amm, 1995; 1998, Sillanpää, 2002), based on measurements of ground magnetic and ionospheric electric fields. We will use a version of the procedure named the one-dimensional (1-D) method of characteristics (Inhester et al., 1992; Sillanpää, 2002), in which we assume 1-D conditions, i.e. vanishing gradients along a certain direction within the region of investigation. A detailed description is given in Sect. 2.2. While the former method provides data on a large-scale resolution $(\sim 800 \mathrm{~km})$, integrated over typically $5 \mathrm{~min}$, the 1-D method of characteristics operates with mesoscale resolution $(\sim 50 \mathrm{~km})$ and having values every $20 \mathrm{~s}$. A comparison between the two methods requires that individual conductances from the latter technique must be averaged. The main objective of this paper is to investigate whether simple averaging of the mesoscale results allows a continuous transition to the large-scale results. Such a transition is not obvious regarding the two sets of conductances as results of stochastical processes operating on different scales. Using data from three periods with substorm activity in 1998, we have examined the relationship between the conductances resulting from the two methods to see if the results converge when averaged over the same spatial and temporal scales. Results are presented and discussed in Sect. 3. A summary is given in Sect. 4, while Sect. 5 provides the conclusions of this study.

\section{Deriving ionospheric conductances}

2.1 Technique 1: Remote sensing from space of UV and $\mathrm{X}$-ray emissions

Through remote sensing of UV and X-ray emissions from space, the precipitating electron energy spectra can be derived. By then estimating height profiles of the resulting ionization, ionospheric Hall and Pedersen conductances are obtained. A detailed description of this method is given by Aksnes et al. (2002; 2004), as well as a discussion of its limitation (Aksnes et al., 2004). Here we just give a brief description of the method.

The UVI camera (Torr et al., 1995) on the Polar satellite measures UV-emissions within the Lyman-Birge-Hopfield (LBH) band (140-180 nm). These UV-emissions are separated into LBHS (140-160 $\mathrm{nm}$ ) and LBHL (160-180 nm). As the shortest wavelengths (LBHS) are subject to considerably greater absorption in the atmosphere than the longer wavelengths (LBHL), we can derive the average electron energy from the ratio between the intensities of the two LBH-bands. We can further calculate the electron energy flux from the intensity of the LBHL emissions. To accumulate the two LBH images needed to perform an electron energy determination from UVI, it takes on the order of two minutes, depending on the operating sequence. UVI has a nominal spatial resolution of $\sim 40 \mathrm{~km}$ from apogee (Torr et al., 1995). The spatial resolution is degraded in one direction to $\sim 360 \mathrm{~km}$ due to wobbling of the Polar satellite. More details about the energy determination from UVI are given by Germany et al. (1997; 1998a,b).

The PIXIE camera (Imhof et al., 1995) measures bremsstrahlung in the energy range of $\sim 2-22 \mathrm{keV}$, produced when precipitating electrons interact with the nuclei of atmospheric particles. The X-ray photons detected by PIXIE go through a 4.4-mm pinhole, leaving us with a resolution in the X-ray source region of $\sim 600-900 \mathrm{~km}$ (depending on Polar altitude). As described by Østgaard et al. (2000; 2001), the X-ray measurements combined with a look-up table produced by a coupled electron-photon transport code (Lorence, 1992) can be used to derive a four-parameter representation of the precipitating electrons from $\sim 3 \mathrm{keV}$ to $\sim 100 \mathrm{keV}$. These electron spectra are typically 4.5 -min averages every $10 \mathrm{~min}$.

The energy characteristics derived from UVI and PIXIE measurements can be combined to give the electron energy distribution from $\sim 0.1$ to $\sim 100 \mathrm{keV}$ (Østgaard et al., 2001), with the temporal and spatial resolution of the PIXIE instrument (4.5 min and $600-900 \mathrm{~km}$ ). An upper limit of modelling errors when deriving mean energies from UVI measurements is estimated to be $23 \%$ (Germany et al., 2001). Comparing the energy spectra derived from UVI and PIXIE data with insitu measurements from the Defense Meteorological Satellite Program (DMSP) satellites, Østgaard et al. (2001) found an average ratio of $1.03 \pm 0.6$ between calculated and measured energy flux from $0.09 \mathrm{keV}$ to $30 \mathrm{keV}$. One possible explanation of the large standard deviation may be the different spatial resolutions.

To calculate the Hall and Pedersen conductances a similar scheme as described by Aksnes et al. $(2002 ; 2004)$ is applied. The main building block of this scheme is the computer code developed by the University of Maryland from the original TANGLE code (Vondrak and Baron, 1976; Vondrak and Robinson, 1985). The input parameters to the code are the same as were used by Aksnes et al. (2002; 2004), except for the collision frequencies. In this study the electron-neutral 


\section{MIRACLE}

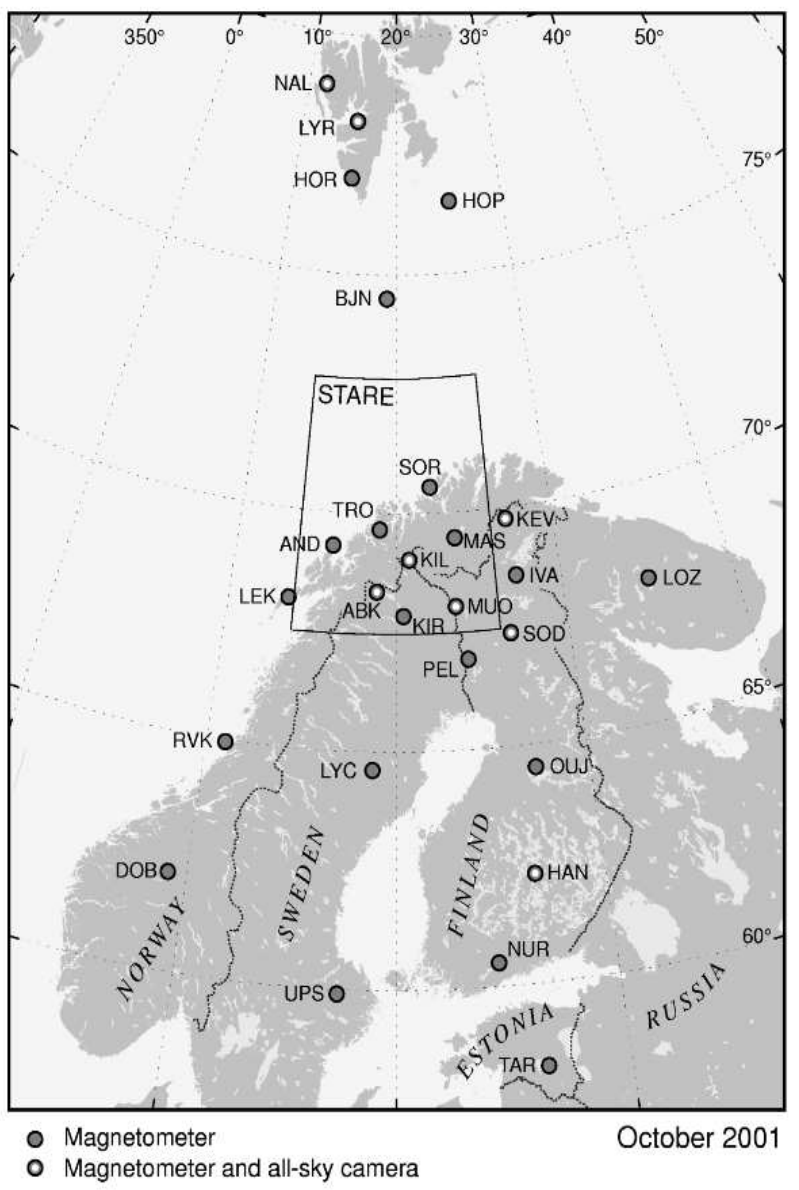

Fig. 1. Map of the MIRACLE network.

collision frequencies are estimated from Itikawa (1971) and Gagnepain et al. (1977), while the ion-neutral collision frequencies are derived using work by Mason (1970), Pesnell et al. (1993), and Viehland and Mason (1995).

\subsection{Technique 2: 1-D method of characteristics}

The ionospheric Hall and Pedersen conductances, $\Sigma_{H}$ and $\Sigma_{P}$, can be derived using measurements of the ionospheric electric field $\boldsymbol{E}$ and the ground magnetic field $\boldsymbol{B}$. The method is called the method of characteristics, as it solves a differential equation along its characteristics. In this study, we will apply this technique using electric field measurements from the Scandinavian Twin Auroral Radar Experiment (STARE) and ground magnetometer data from the International Monitor for Auroral Geomagnetic Effects (IMAGE) network.

A map showing the Magnetometers - Ionospheric Radars - Allsky Cameras Large Experiment (MIRACLE) network is presented in Fig. 1. The IMAGE magnetometer sites are indicated as dots, while the STARE field of view (FOV) is marked by the black rectangle. The method of characteristics is described in detail by Inhester et al. (1992), Amm (1995; 1998), and Sillanpää (2002). Here, we will only give a general overview, including the most important equations. Note that we will use the 1-D version of the procedure, assuming that gradients along a certain direction are vanishing within the region of investigation. We will first introduce the equations related to the two-dimensional (2-D) method of characteristics, and then proceed to the $1-\mathrm{D}$ version.

When using the method of characteristics, the ionosphere is considered an infinitely thin conducting layer $100 \mathrm{~km}$ above the Earth's surface, in which the Hall and Pedersen currents are flowing. The first step is to extract the external part of the ground magnetic field disturbance. A field continuation to the ionosphere is then performed (Mersmann et al., 1979; Untiedt and Baumjohann, 1993), and we derive the ionospheric equivalent current $\boldsymbol{J}_{\text {eq,ion }}$. According to Fig. 1 the ground magnetometer data cover only about half the area of STARE. Pulkkinen et al. (2003) have shown, though, that the equivalent currents can be reliably reconstructed in the whole STARE FOV, including those areas which are not in an immediate vicinity of the ground magnetometers.

The true ionospheric current $\boldsymbol{J}$ can be separated into a curl-free component $\boldsymbol{J}_{c f}$ and a divergence-free component $\boldsymbol{J}_{d f}$ (Duschek and Hochrainer, 1961). While $\boldsymbol{J}_{c f}$ is associated with the field-aligned current $j_{z}$ above the ionosphere, $\boldsymbol{J}_{d f}$ is related to the horizontal component of the external magnetic field perturbation $\left(\boldsymbol{B}_{e}\right)_{h}$ immediately below the ionosphere (Untiedt and Baumjohann, 1993). Note that the subscript $h$ indicates the horizontal component of $\boldsymbol{B}_{e}$. By assuming that the Earth's geomagnetic field lines are directed vertically downward into the $\hat{z}$ direction, $\boldsymbol{J}_{d f}$ will completely determine $\left(\boldsymbol{B}_{e}\right)_{h}$ (Bostrøm, 1964; Vasuliunas, 1970; Fukushima, 1976). If the angle $\chi$ between the ionospheric plane and the geomagnetic field lines deviates from $90^{\circ}$, then there is also some dependence of $\left(\boldsymbol{B}_{e}\right)_{h}$ on $\boldsymbol{J}_{\boldsymbol{c} f}$ (Fukushima, 1976). At auroral latitudes, though, it is appropriate to disregard the tilting between the Earth's magnetic field with respect to the vertical. The conductance calculations using a typical value of $\chi=77^{\circ}$ for the measuring sites in Northern Scandinavia to be compared with $\chi=90^{\circ}$ have been examined by Amm (1995) during four different situations: a two-dimensional eastward electrojet, a Harang discontinuity, an omega band and a westward travelling surge. The investigation by Amm (1995) revealed, with a few exceptions, only minor differences of a few $\%$ for most of the examined areas.

Another basic electrodynamic equation needed when using the method of characteristics is Ohm's law. Here we disregard possible effects of the neutral wind (Untiedt and Baumjohann, 1993). We further assume a ratio $\alpha$ between the Hall and Pedersen conductances to be given:

$\alpha=\frac{\Sigma_{H}}{\Sigma_{P}}$.

Sillanpää (2002) has studied the dependence of conductances on $\alpha$ when using the 1-D method of characteristics, and concludes that assuming a wrong $\alpha$ will not have a significant affect on the calculated Hall conductance. The Pedersen conductance is more dependent on $\alpha$, as this parameter is calculated directly from Eq. (3). In this study, we use the con- 
ductance calculations from UVI and PIXIE (see Sect. 2.1) to determine $\alpha$.

By combining all the relations established, we can provide a formula giving the Hall conductance $\Sigma_{H}$ along a characteristic $\boldsymbol{r}(\ell)$, which is a line in the xy-plane:

$$
\begin{aligned}
& \Sigma_{H}(\boldsymbol{r}(\ell))=\Sigma_{H}\left(\boldsymbol{r}_{0}\right) \exp [-I(0, \ell)] \\
& \quad+\int_{0}^{\ell} \frac{D(\boldsymbol{r}(\ell))}{|\boldsymbol{V}(\boldsymbol{r}(\ell))|} \exp \left[-I\left(\ell^{\prime}, \ell\right)\right] d \ell^{\prime}
\end{aligned}
$$

with

$$
\begin{aligned}
& I\left(\ell^{\prime}, \ell\right)=\int_{\ell^{\prime}}^{\ell} \frac{C\left(\boldsymbol{r}\left(\ell^{\prime \prime}\right)\right)}{\left|\boldsymbol{V}\left(\boldsymbol{r}\left(\ell^{\prime \prime}\right)\right)\right|} \\
& \boldsymbol{V}=\boldsymbol{E}-\frac{\hat{z} \times \boldsymbol{E}}{\alpha} \\
& C=\nabla_{h} \cdot \boldsymbol{V} \\
& D=\frac{2}{\mu_{0}} \nabla_{h} \cdot\left(\boldsymbol{B}_{\boldsymbol{e}}\right)_{h} .
\end{aligned}
$$

Equation (4) represents the two-dimensional method of characteristics, as $\boldsymbol{r}(\ell)$ lies on the xy-plane. The problem can be much simplified, though, if we assume a one-dimensional situation for which the gradient of all quantities vanishes in one horizontal direction (Inhester et al., 1992; Sillanpää, 2002). By taking $\frac{\delta}{\delta y}=0$ and $\frac{\delta \ell}{\delta x}=\frac{|\boldsymbol{V}|}{V_{x}}$, we can replace Eqs. (5), (7) and (8) with:

$I\left(\ell^{\prime}, \ell\right)=\ln \left(\frac{V_{x}(x)}{V_{x}\left(x^{\prime}\right)}\right)$

$C=\frac{\delta V_{x}}{\delta x}$

$D=\frac{2}{\mu_{0}} \frac{\delta B_{e, x}}{\delta x}$.

Insertion of Eqs. (9), (10) and (11) into Eq. (4) yields the 1-D method of characteristic solution for the Hall conductance :

$\Sigma_{H}(x)=\frac{\frac{2}{\mu_{0}} B_{e, x}+K}{V_{x}(x)}$

The constant $K$ in Eq. (12) represents the effect of distant currents. If for some $x_{0}, V_{x}\left(x_{0}\right)=0$ holds, $K$ is uniquely defined. In other cases we use the criterion that at the edges of the electrojet region the conductances drop to a given background value (see Inhester et al., 1993; Sillanpää, 2002) to infer $K$.

In this study, we have used the 1-D method of characteristic to derive the ionospheric Hall and Pedersen conductances in the Northern Scandinavian region, having a spatial resolution of $\sim 50 \mathrm{~km}$ and a temporal resolution of $\sim 20 \mathrm{~s}$. As the procedure requires that the electrodynamical parameters only change in one direction within the STARE FOV, investigations have been performed to check that the different time steps and regions involved satisfy the 1-D condition. This means that all quantities should have a vanishing derivative along a certain direction. In our case we assume that this direction is zonal (east-west), while we allow for variations in the meridional direction. The criterion for using the 1-D method is that the changing length of a parameter should be larger than the length of the relevant analysis area in the east-west direction. If the parameter is $X$, then the changing length is defined as $L=\left|X /\left(\frac{d X}{d L}\right)\right|$. The electric field strength must be more than $\sim 17 \mathrm{mV} / \mathrm{m}$ for STARE to provide reliable results (Sillanpää, 2002). For each time step along each latitude for which sufficient STARE data are available, we check whether the electric field is one-dimensional. Regions where this is not the case are excluded from the conductance calculation. Another possibility is that the conductances themselves do not satisfy a 1-D condition. This situation will in most cases, but not all, cause a considerable non-1-D ground magnetic field. Such cases are more difficult to reveal, due to the limited east-west coverage of magnetometer stations within the STARE region (see Fig. 1). A more thorough discussion about this problem is provided in Sect. 3 when interpreting the conductance profiles established using the two different techniques.

As shown in this section, the Hall conductance is the primary output from the 1-D method of characteristics, from which other quantities like, for example, field-aligned currents (FACs) can be calculated. In a recent study by Amm et al. (2003), the FACs calculated using the 1-D method of characteristics are compared with FAC derived from in-situ measurements by the Cluster satellites. By showing a convincing agreement between the two sets of FAC data, Amm et al. (2003) indirectly demonstrate that the Hall conductances derived using the 1-D method of characteristics give reliable results.

\subsection{Assumptions and limitations of the two techniques}

As explained in Sect. 1, the main objective of this paper is to investigate whether simple averaging of mesoscale results (1-D method of characteristics) allows for a continuous transition to large-scale results (the UVI/PIXIE analysis). While the latter technique operates with a resolution of $\sim 800 \mathrm{~km}$ in space and $\sim 4.5 \mathrm{~min}$ in time, the ground-based method establishes conductances every $20 \mathrm{~s}$ and with a spatial resolution of $\sim 50 \mathrm{~km}$. This comparison is further complicated by several different assumptions and limitations of the two techniques.

When using the 1-D method of characteristics, we assume 1-D conditions (see Sect. 2.2). This is probably the most crucial assumption regarding the ground-based technique. It is not straightforward to identify whether the conductances satisfy 1-D conditions. This will be subject for investigations in the following Sect. 3. Regions where $\boldsymbol{E}$ is non 1-D can be fairly easily identified and excluded from the calculations. Such cases will therefore not affect the average Hall and Pedersen conductances derived by the ground-based method. On the other hand, the same regions will still contribute to the conductances using the UVI/PIXIE technique. A similar 

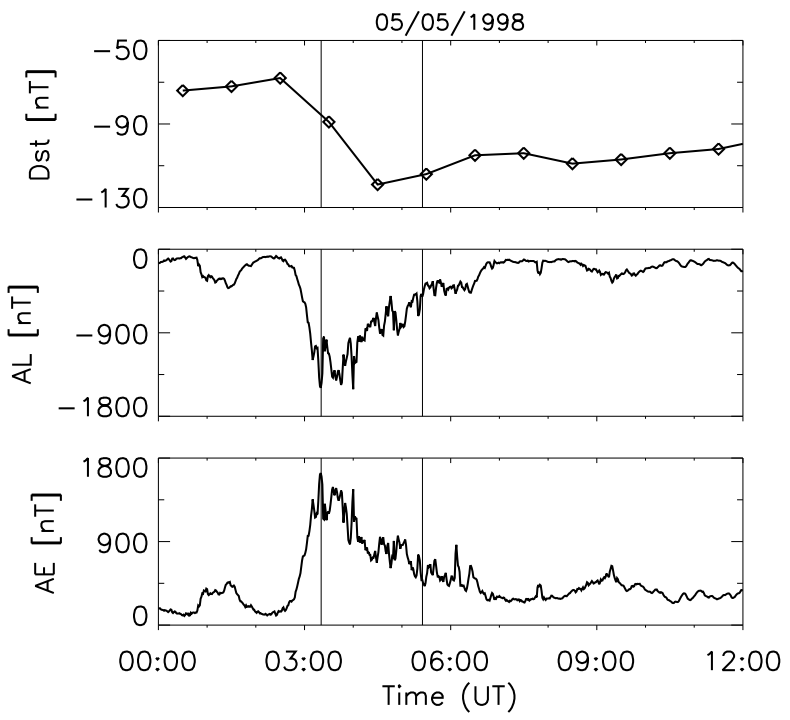

Fig. 2. The AE, AL and $D_{s t}$-indices between 00:00 and 12:00 UT on 5 May 1998. The two vertical solid lines indicate our time interval with data between 03:20 and 05:25 UT.

problem occurs when STARE data are unable to provide a reliable electric field value.

As pointed out in Sect. 2.1, the UVI data are responsible for the lower part of the precipitating electron energy spectra up to $\sim 15-20 \mathrm{keV}$. The estimation of the electron energies using UVI measurements within time periods of $\sim 4.5 \mathrm{~min}$ may be affected in two ways. First of all, the LBHL and LBHS images are not coincident as they are measured during different time intervals of $37 \mathrm{~s}$. In cases where the auroral morphology is significantly changing between the two observations, this leads to an analysis problem, though the uncertainty is difficult to estimate. A second problem is that the UVI measurements are not continuous within the investigated time intervals of $\sim 4.5 \mathrm{~min}$. For example, it takes $1 \mathrm{~min}$ and $51 \mathrm{~s}$ to perform a full energy analysis from UV-emissions for the operating modes used in this study. Then there is a gap of $1 \mathrm{~min}$ and $13 \mathrm{~s}$ before the procedure is repeated. If sudden changes in the particle precipitation takes place during such gaps, this will complicate the comparison with the 1-D method of characteristics.

The resolution of the PIXIE instrument is on the order of $\sim 600-900 \mathrm{~km}$ (see Sect. 2.1), depending on the altitude of the Polar satellite. In comparison, the STARE FOV is nominally approximately $470 \mathrm{~km} \times 540 \mathrm{~km}$, as it covers a geographic region of 67.1-72.0 in latitude and 14.0-26.0 in longitude. For two of the three events studied in this paper, the most equatorward region has been left out of the analysis, due to a very limited number of ion velocity vectors produced by the two radar beams. This reduces the latitudinal width from $540 \mathrm{~km}$ to $380 \mathrm{~km}$. The inconsistency between the PIXIE's resolution and the STARE's FOV leads to another source of uncertainty which is discussed in more detail in Sect. 3.

\subsection{Selection of data}

Three periods with substorm activity in 1998 have been selected for this study. The two events of 5 May and 26 June 1998, include data from parts of the substorm expansion phase and most of the recovery phase. For the 12 August 1998 event, we have measurements from the start of substorm onset until the beginning of the recovery phase.

A number of only three events for a comparison study may seem low, but is due to a variety of reasons. First of all, to use the 1-D method of characteristics, the STARE radars must receive enough backscatter to estimate the electric field. Most of the time STARE sees no echoes at all. Either there are too few electrons in the critical altitude range, or the electric field is too weak to form irregularities. While the former imply that substorm periods should be most suited for studies using STARE, the latter suggest that problems may sometimes occur during the substorm expansion phase, when the electron density and therefore also the conductances can be extremely high in some small volume. If the magnetosphere acts as a current generator, this can lead to a suppression of the electric field to very small values below the threshold of the radars. In general, though, STARE is well suited to investigate the electrodynamics during substorms, and so are also instruments performing remote sensing from space. A comparison between the two techniques, however, requires that the Polar satellite for the time period of interest is located above the Northern Hemisphere, with the UVI and PIXIE instruments viewing the Scandinavian region. Substorm conditions are best suited, as we need to detect sufficient X-ray photons by PIXIE to derive a proper electron spectrum. The UVI camera, which can operate in different modes to investigate certain aspects of the auroral activity, must further operate in a special mode if a full energy analysis is to be performed. The most critical requirement limiting the number of possible events, though, is the fact that we have only considered the date period between May and September, 1998. While the Finnish STARE radar station Hankasalmi suffered from hardware problems in 1997 and the beginning of 1998, the PIXIE chamber detecting X-ray photons between $\sim 8-22 \mathrm{keV}$ was no longer functioning after 30 September 1998. PIXIE continued to detect X-ray photons between $\sim 2-8 \mathrm{keV}$ until November 2002, but the lack of information about the most energetic X-rays strongly increased the uncertainty in estimating the high energy tail of the precipitating electrons up to $100 \mathrm{keV}$ affecting the Hall conductance (Aksnes et al., 2002; 2004).

\section{Results and discussion}

\subsection{May 1998}

In Fig. 2 we present the geomagnetic indices $\mathrm{AE}, \mathrm{AL}$ and $D_{s t}$, revealing a substorm expansion phase commencing around 03:00 UT. At 03:21 UT, when the AE- and ALindices reach their largest values of $1638 \mathrm{nT}$ and $-1493 \mathrm{nT}$ 
respectively, the $D_{s t}$-diagram show that we are in the main phase of a geomagnetic storm. A minimum in $D_{s t}$ of $-119 \mathrm{nT}$ between 04:00 and 05:00 UT means this event is classified as an intense storm (Gonzalez et al., 1994). We have estimated the conductances between 03:20 and 05:25 UT, as indicated by the two vertical solid lines. This time interval includes parts of the expansion phase and then the recovery phase.

The results of the conductance calculations using the two techniques including remote sensing of UV and X-ray emissions (see Sect. 2.1) and the 1-D method of characteristics (see Sect. 2.2) are presented in Fig. 3. The solid lines in Figs. $3 \mathrm{a}$ and $\mathrm{b}$ indicate the Hall and Pedersen conductances derived from the UVI/PIXIE analysis, while the dashed lines represent the conductances using the 1-D method of characteristics. The conductance values are $10 \mathrm{~min}$ apart, with an integration time of about $4.5 \mathrm{~min}$. For this event, the STARE radar measurements are taken from a geographic region of 67.1-72.0 in latitude and 14.0-26.0 in longitude, meaning that the STARE FOV is approximately $470 \mathrm{~km} \times 540 \mathrm{~km}$.

In the beginning of the investigated time interval, we observe differences between the two conductance profiles in both magnitude and trend. The values derived using the 1-D method of characteristics show most variability, with a maximum Hall conductance of $33 \mathrm{~S}$ at 04:00-04:05 UT and a minimum $20 \mathrm{~min}$ later of only $11 \mathrm{~S}$. For this time step, the conductances using ground-based measurements are more than $60 \%$ less than the values derived from remote sensing of UV and X-ray emissions. This is clearly indicated by the plot in Fig. 3c, showing the differences in \% when calculating the conductances using the 1-D method of characteristics instead of using UVI/PIXIE data. As explained in Sect. 2.2, the Pedersen conductance calculated using the 1-D method of characteristics depends on the ratio $\alpha$ between the Hall and the Pedersen conductances derived from the UVI/PIXIE analysis. Consequently, the percentages given in Fig. $3 \mathrm{c}$ correspond to both the differences between the two Hall conductance sets in Fig. 3a, as well as the differences between the two Pedersen conductance sets in Fig. $3 \mathrm{~b}$.

In the next time frame of 04:30-04:35 UT, a shear region intrudes into the STARE FOV. This causes a considerable dropout of STARE data, and therefore this time step is left out in the analysis. For the rest of the time period from 04:40 until 05:25 UT, the two conductance profiles match fairly well, except for the time step of 04:50-04:55 UT.

As described in Sect. 2.3, the different assumptions and limitations of the two techniques imply that a meaningful comparison relies strongly on the level of turbulence of the ionosphere. We have therefore developed a method to separate the data points into relatively uniform conditions and non-uniform conditions. Relatively uniform conditions are considered to be present if the following three selection criteria are fulfilled: 1) More than $70 \%$ of the individual magnetic scale lengths must be larger than $429 \mathrm{~km}$. 2) The largest UVIinhomogeneity within the STARE FOV must be less than $100 \%$. 3) The difference between the average UVI intensity within the STARE FOV vs. a larger region of $\sim 800 \mathrm{~km}$

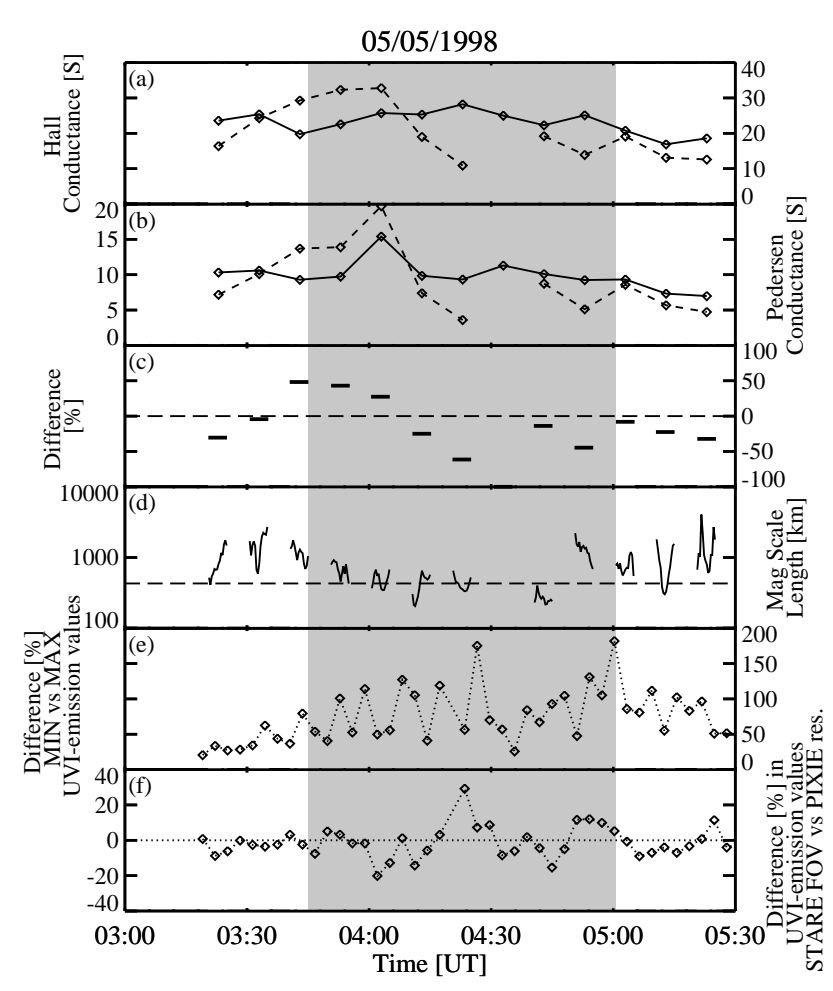

Fig. 3. (a) The Hall and (b) Pedersen conductances [S] derived in the Scandinavian region using UVI and PIXIE measurements (solid line) and the 1-D method of characteristics (dashed line) on 5 May 1998. (c) The difference in \% between the two conductance sets. The horizontal dashed line is drawn to give the 0-percent level, while the length of the lines indicates the integration time period. (d) Magnetic field scale lengths $(\mathrm{km})$ between the two MIRACLE magnetometer stations of AND and KEV. (e) The average percentage calculated using the lowest and largest UVI-LBHL emission values at five strips of constant latitude within the STARE FOV. (f) The difference in $\%$ for the average UVI-LBHL intensity within the STARE FOV compared with the average UVI-LBHL intensity when including a larger area surrounding the STARE FOV matching the PIXIE resolution.

(the PIXIE resolution) must be less than 20\%. A detailed description of these three selection criteria will be given in the following when discussing Fig. 3.

The first selection criterion involves the term magnetic scale lengths. As explained in Sect. 2.2, 1-D conditions are required for the different parameters when using the 1-D method of characteristics. The electric field data can be tested most efficiently, meaning that regions where $\boldsymbol{E}$ is not 1-D are excluded from the conductance calculation. To decide whether the conductances themselves satisfy a 1-D condition is more difficult. Since such a situation most often results in a non-1-D ground magnetic field, the latter can be investigated. The problem is the limited east-west coverage of magnetometer stations in the STARE region. When calculating the conductances using the 1-D method of characteristics, an investigation is performed for the conditions between the two MIRACLE magnetometer stations of Andenes (AND) 

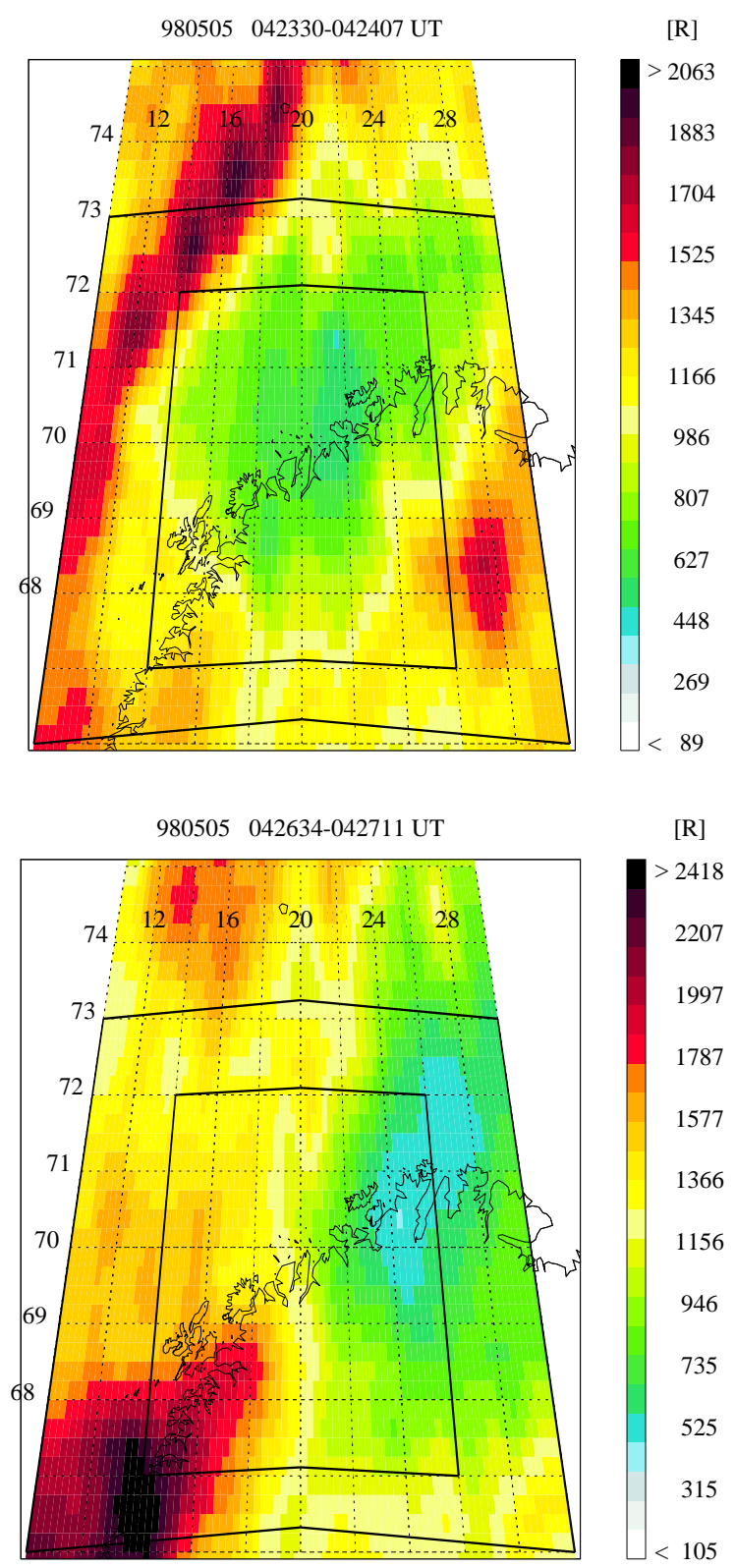

Fig. 4. The UVI-LBHL images for the two time steps of (a) 04:23:30-04:24:07 UT and (b) 04:26:34-04:27:11 UT on 5 May 1998. The inner black box drawn on top of the images corresponds to the STARE FOV, while the outer box matches the PIXIE resolution of $\sim 800 \mathrm{~km}$.

and Kevo (KEV), which are located at almost the same geographic latitude ( $\sim 69.3$ and $\sim 69.8^{\circ}$, respectively). When the calculated magnetic scale lengths are smaller than a heuristical limit of $\sim 429 \mathrm{~km}$ (the extent of the east-west chain AND$\mathrm{KEV}$ ), this means we may not have a 1-D condition. For such a situation, we should be careful when interpreting the conductances using the 1-D method of characteristics. All data within the STARE FOV are still included in the conductance calculation, as the investigation performed only involves the conditions between AND and KEV. Other regions within the STARE FOV are not investigated, as the limited east-west coverage of magnetometer stations prevents an efficient check similar to the procedure regarding the electric field.

In Fig. 3d, we present magnetic scale lengths estimated between AND and KEV for the different time periods. The scale lengths are calculated every $20 \mathrm{~s}$, and we plot the individual values in $\mathrm{km}$ as solid lines for the different time steps. We observe that the values are usually larger than the heuristical limit of $\sim 429 \mathrm{~km}$ shown by a dashed horizontal line, indicating that our assumption of a 1-D condition holds. During some time intervals, though, many of the individual scale lengths drop below $429 \mathrm{~km}$, suggesting more disturbed conditions. Our first selection criterion requests that more than $70 \%$ of the individual magnetic scale lengths (in the $\sim 5$ min time frames) must be larger than the heuristical limit of $429 \mathrm{~km}$. This is fulfilled for the first four time steps, as well as the last four. During the period 04:00-04:45 UT, however, the investigation of magnetic scale lengths indicates too much ionospheric turbulence.

The second selection criterion also deals with the necessary 1-D condition for the 1-D method of characteristics. We have used UVI-LBHL measurements to monitor changes in precipitated energy flux. First, we have divided the STARE FOV into a $5 \times 4$ grid, with each subregion being $1^{\circ}$ in latitude and $3^{\circ}$ in longitude. Then we have calculated the differences in \% between the lowest and the largest UVI intensities along each of the five strips of constant latitude. Based on these five individual values derived for the latitude sectors of $67-68^{\circ}$, $68-69^{\circ}, 69-70^{\circ}, 70-71^{\circ}$, and $71-72^{\circ}$ respectively, an average percentage value has been established for each UVI data set, having a temporal resolution of $37 \mathrm{~s}$. For simplicity, we will refer to such values as "UVI-inhomogeneities" throughout the text.

The results are presented in Fig. 3e. In the beginning of the time interval, the UVI-inhomogeneities are usually less than $50 \%$. The first minor intensification is found at 03:34 UT, when the value reaches $62 \%$. Throughout the next hour, we observe an increasing trend of the UVI-inhomogeneities, coinciding with a reduction of the magnetic scale lengths presented in Fig. 3d. Between $\sim 04: 00$ and 04:30 UT, the UVIinhomogeneities sometimes exceed $100 \%$ while the scale lengths drop below $\sim 429 \mathrm{~km}$.

In Figs. 4a and b, we have plotted the UVI images for the two time steps of 04:23:30-04:24:07 UT and 04:26:3404:27:11 UT. The inner black box drawn on top of each image indicates the STARE FOV, while the outer box corresponds to a region of $\sim 800 \mathrm{~km}$, matching the PIXIE resolution. Note that the colors in the two plots correspond to different intensities, as the dark red color is set equal to the maximum value in each plot.

The latter image presented in Fig. 4b reveals highly nonuniform conditions in the east-west direction. At the eastern border, the UVI-intensities are small, as indicated by the blue and green color. On the contrary, the UVI-intensities are much larger at the western border, shown by the red and orange color. According to Fig. 3e, the average UVIinhomogeneity for this time step reaches almost $200 \%$. 
Such variability in the east-west direction complicates the calculation of conductances when using the 1-D method of characteristics. Our second selection criterion to identify relatively uniform conditions requires that the UVIinhomogeneities must be below $100 \%$. This means that the time steps of $\sim 03: 50-03: 55$ and $\sim 04: 50-04: 55$ UT should also be assigned non-uniform conditions, in addition to the time period of $\sim 04: 00-04: 45$ already determined from the previous investigation of magnetic scale lengths (Fig. 3d).

The third selection criterion deals with the difference between the STARE FOV and the much coarser PIXIE resolution. From the image given in Fig. $4 \mathrm{a}$, we note a significant UVI intensity surrounding the STARE region. For this event on 5 May 1998, the PIXIE resolution of $\sim 800 \mathrm{~km}$ corresponds to an area about 2.5 times larger than the STARE FOV of $470 \mathrm{~km} \times 540 \mathrm{~km}$. The inconsistency between the PIXIE resolution and the STARE's FOV implies that the conditions in the particle precipitation outside the STARE region may influence the PIXIE measurements and therefore affect the conductance calculation. This possible source of error has been studied by investigating the UVI-LBHL emissions in a region larger than the actual analyzing area. The much better spatial and temporal resolution given by the UVI camera provides us with a tool to investigate the reliability of the PIXIE data. First, we have calculated the average UVI-LBHL intensity within the STARE FOV, a region corresponding to the inner black box in Fig. 4. Then we have repeated the calculation and taken into account a larger area surrounding the STARE FOV matching the PIXIE resolution. This larger region corresponds to the outer black box in Fig. 4 .

In Fig. 3f, we present the differences in $\%$ between the UVI intensities derived using the two approaches. Negative values mean that the average UVI intensity is largest within the STARE FOV. Since regions with less precipitation surrounding the STARE FOV matching the PIXIE resolution also influence the X-ray data, we may underestimate the conductances using UVI/PIXIE data during such situations. Likewise, positive values in Fig. $3 \mathrm{f}$ indicate stronger particle precipitation outside the region of investigation and a possible overestimation of the conductances when using the satellite data. By comparing the results in Figs. $3 \mathrm{c}$ and $\mathrm{f}$, we note some resemblance between the values. Around $\sim 04: 00$ UT, we observe the lowest value in Fig. 3f of less than $-20 \%$. At this time the conductances using the 1-D method of characteristics are almost 30\% larger than the conductances using UVI/PIXIE data. Then the situation is reversed around 04:23 UT. The 1-D values are more than $60 \%$ less than the satellite calculations and Fig. $3 \mathrm{f}$ reveals a positive value of about $30 \%$. Our third selection criterion states that the difference between the average UVI intensity for a region corresponding to the STARE FOV vs. a larger region of $\sim 800 \mathrm{~km}$ (the PIXIE resolution) must be below $20 \%$. This means that the two time steps of 04:00-04:05 and 04:20-04:25 UT must be classified as periods when the ionosphere is in a highly turbulent state. Both time steps have already been assigned non-uniform conditions, though, based on the previous analysis of magnetic scale lengths and UVI-inhomogeneities.
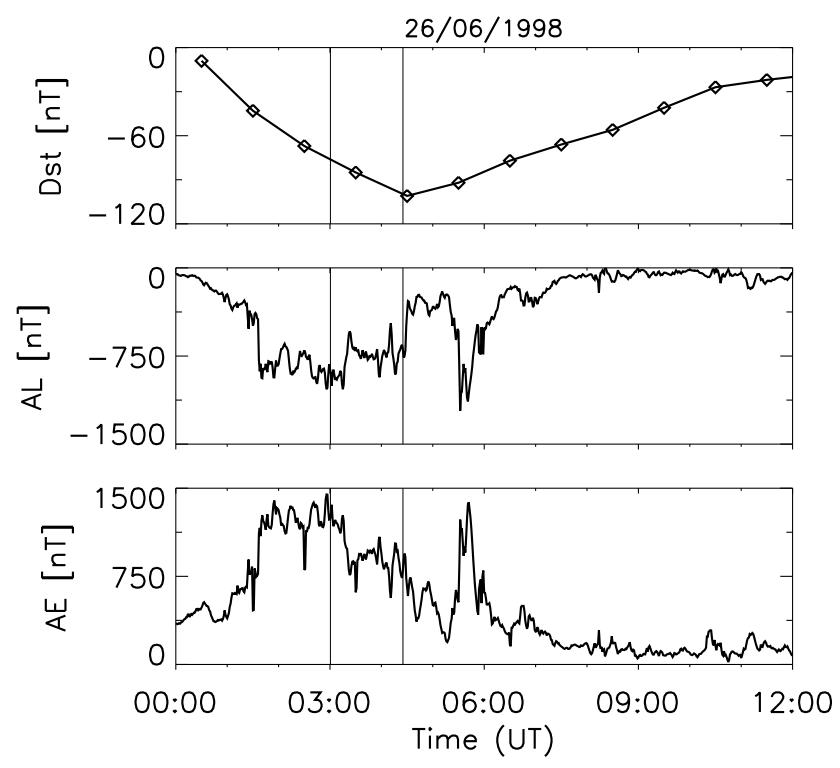

Fig. 5. The AE, AL and $D_{s t}$-indices between 00:00 and 12:00 UT on 26 June 1998. The two vertical solid lines indicate our time interval with data between 03:00 and 04:15 UT.

By performing several investigations and requiring that certain selection criteria associated with each of them are fulfilled, we are able to identify time periods when a better match between the two conductance sets should be expected. Using different methods is helpful, as each procedure has its advantages as well as disadvantages. For example, the magnetic scale lengths are estimated continuously, while the UVI-inhomogeneities are calculated every 2 min with an integration time of $37 \mathrm{~s}$. While this suggests that it should be sufficient to only deal with the former, the magnetic scale lengths have a limited spatial coverage compared with UVI. Consequently, the latter procedure may reveal disturbed conditions not captured by the analysis of magnetic scale lengths.

To distinguish between the data points during relatively uniform conditions and non-uniform conditions, the latter has been marked with a dark grey shading in Fig. 3. For 5 of the 6 time steps when all three selection criteria are fulfilled, the differences between the two conductance sets are less than $\pm 30 \%$. On the contrary, only 3 of the 6 time steps during non-uniform conditions between $\sim 03: 45$ and 05:00 UT reveal conductances within $\pm 30 \%$.

\subsection{June 1998}

The geomagnetic indices $\mathrm{AE}, \mathrm{AL}$ and $D_{s t}$, between 00:00 and 12:00 UT on 26 June 1998, are presented in Fig. 5. We observe a substorm expansion phase taking place around 01:40 UT, followed by a period with strong AE- and ALindices. The largest values are found close to 03:00 UT, with AE reaching $1450 \mathrm{nT}$ and AL droping below $-1000 \mathrm{nT}$. We have measurements between 03:00 and 04:15 UT, corresponding to the end of the expansion phase and most of the 


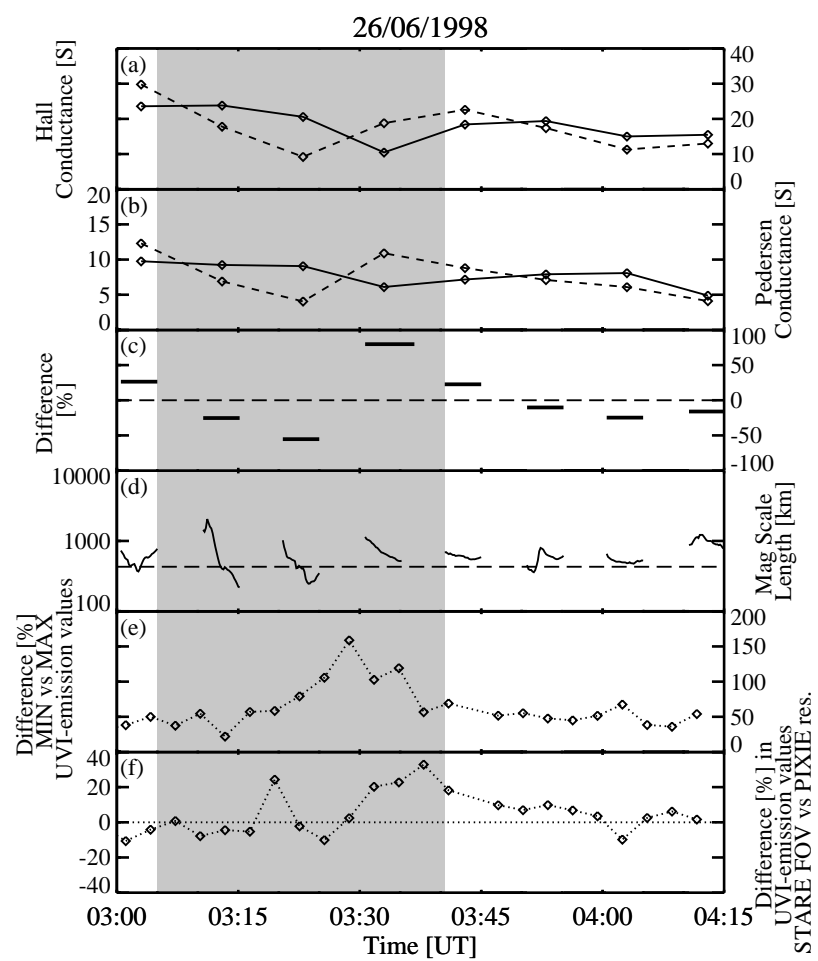

Fig. 6. (a) The Hall and (b) Pedersen conductances [S] derived in the Scandinavian region using UVI and PIXIE measurements (solid line) and the 1-D method of characteristics (dashed line) on 26 June 1998. (c) The difference in \% between the two conductance sets. The horizontal dashed line is drawn to give the 0-percent level, while the length of the lines indicates the integration time period. (d) Magnetic field scale lengths $(\mathrm{km})$ between the two MIRACLE magnetometer stations of AND and KEV. (e) The average percentage calculated using the lowest and largest UVI-LBHL emission values at four strips of constant latitude within the STARE FOV. (f) The difference in $\%$ for the average UVI-LBHL intensity within the STARE FOV compared with the average UVI-LBHL intensity when including a larger area surrounding the STARE FOV matching the PIXIE resolution.

following recovery phase. As the $D_{s t}$-index value reaches a minimum of $-101 \mathrm{nT}$ between 04:00 and 05:00 UT, we further note that we are in the main phase of an intense geomagnetic storm.

In Figs. $6 \mathrm{a}$ and $\mathrm{b}$ we present the calculated Hall and Pedersen conductances similar to Figs. $3 \mathrm{a}$ and $\mathrm{b}$. The STARE radar measurements for this event have a lower latitude limit of $68.6^{\circ}$, resulting in a STARE FOV of about $470 \mathrm{~km} \times 380 \mathrm{~km}$. Highly disturbed ionospheric conditions occur for the three time periods between $\sim 03: 10$ and 03:40 UT, as indicated by the dark grey area in Fig. 6. During the remaining 5 time periods, the ionosphere is in an appropriate state considering our three selection criteria presented in Sect. 3.1.

As seen in Fig. 6c, two of the time intervals, 03:20-03:25 and 03:30-03:35 UT, show differences between the derived conductances of -55 and $80 \%$, respectively. This may be explained by a lack of 1-D conditions. According to Fig. 6d, more than $50 \%$ of the individual magnetic scale length values are below the heuristical limit of $\sim 429 \mathrm{~km}$ between 03:20 and 03:25 UT. We further observe in Fig. 6e that the investigation of UVI intensities along strips of constant latitude within the STARE FOV reveals the largest differences during the time interval of 03:25 and 03:35 UT, varying between $\sim 100$ and $\sim 160 \%$. Consequently, these two time periods of 03:20-03:25 and 03:30-03:35 UT are assigned non-uniform conditions.

The UVI images for the two time steps of 03:10:1803:10:55 UT and 03:31:46-03:32:23 UT are plotted in Figs. 7a and b. Fairly stable conditions in the east-west direction are found in the first image, suggesting that the 1D condition holds for the start of the time step of $\sim 03: 10$ 03:15 UT. This is supported by individual magnetic scale lengths of $\sim 1400 \mathrm{~km}$. After a maximum scale length of more than $2000 \mathrm{~km}$ around 03:11 UT, the values decrease strongly and reach a minimum of $218 \mathrm{~km}$ (Fig. 3d). Only $\sim 50 \%$ of the individual scale lengths are larger than $429 \mathrm{~km}$ within the time frame of $\sim 03: 10-03: 15$ UT, meaning that selection criterion No. 1 is not fulfilled.

Figure $7 \mathrm{~b}$ reveals large variations along the strips of constant latitude, corresponding well with the UVIinhomogeneities in Fig. 6e, exceeding 100\% at these times. Consequently, the time step of $\sim 03: 30-03: 35$ UT is attributed to non-uniform ionospheric conditions. This is also reflected in the investigation of the differences between the average UVI intensity for a region of $470 \mathrm{~km} \times 380 \mathrm{~km}$ (the STARE FOV) and $\sim 800 \mathrm{~km}$ (the PIXIE resolution), respectively. As shown in Fig. 6f, the largest differences of more than 20\% occur between $\sim 03: 20$ and 03:40 UT.

Our analysis of the local ionospheric turbulence for this event on 26 June 1998, shows that non-uniform conditions appear during the three time steps between $\sim 03: 10$ and $\sim 03: 40$ UT. Only 1 of the 3 calculated conductance sets during this time period differs less than $\pm 30 \%$. On the contrary, all 5 time steps when we have relatively uniform ionospheric conditions provide conductances with differences within $\pm 30 \%$.

\subsection{August 1998}

Figure 8 presents the geomagnetic indices $\mathrm{AE}, \mathrm{AL}$ and $D_{s t}$, between 20:00 and 08:00 UT on 12-13 August 1998. In the beginning of the time period, when we have measurements between 23:30 and 23:55 UT on 12 August, we observe a significant increase in the AE- and AL-indices. The largest values are found around 23:40 UT, when AE reaches $\sim 650$ and AL exceeds $-400 \mathrm{nT}$. Then the magnetic activity decreases at the end of the time period, suggesting that our time interval of 23:30-23:55 UT includes the substorm expansion phase and the start of the recovery phase. We further note that no geomagnetic storm is present, as the $D_{s t}$-values are small.

The estimated conductances for the time period with measurements on 12 August 1998, are presented in Figs. $9 \mathrm{a}$ and b. The STARE FOV is $\sim 470 \mathrm{~km} \times 380 \mathrm{~km}$, 
(a)

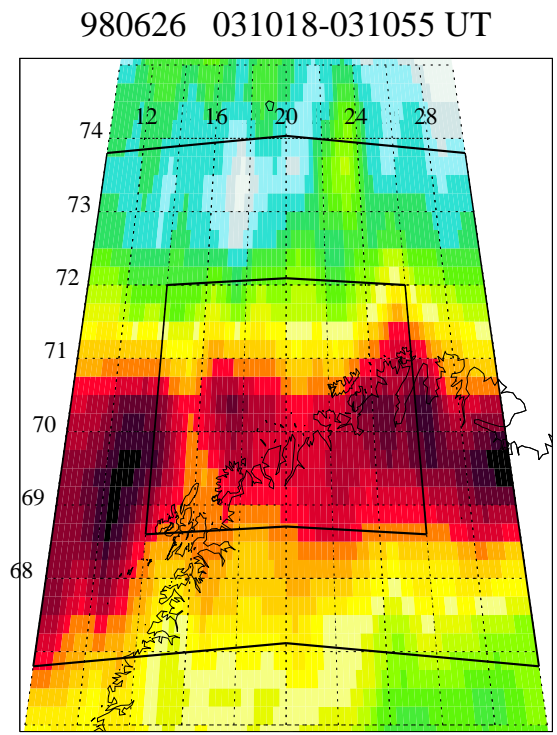

(b)

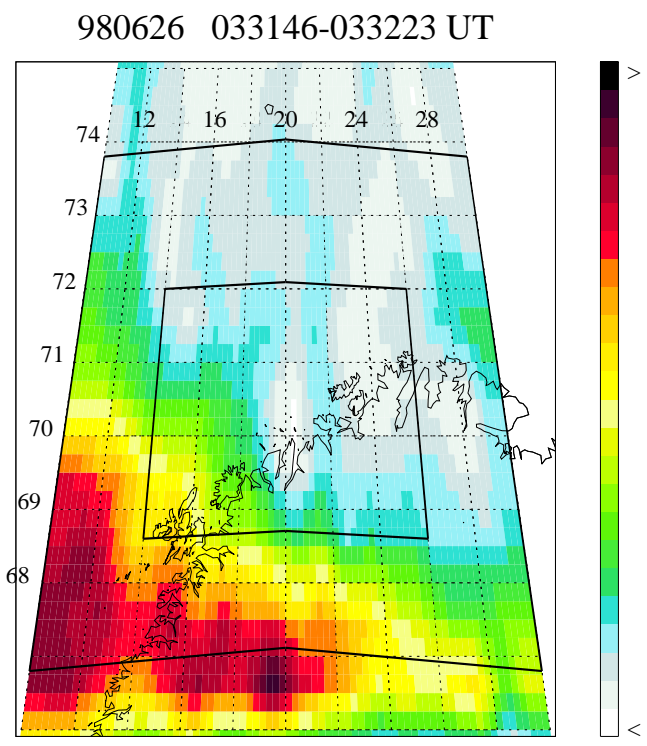

[R]

1488

1358

1229

1099

970

841

711

582

452

323

194

64

Fig. 7. The UVI-LBHL images for the two time steps of (a) 03:10:18-03:10:55 UT and (b) 03:31:46-03:32:23 UT on 26 June 1998. The inner black box drawn on top of the images corresponds to the STARE FOV, while the outer box matches the PIXIE resolution of $\sim 800 \mathrm{~km}$.

similar to the event on 26 June 1998. The two conductance profiles are much alike. Both the magnetic scale lengths (Fig. 9d) and the UVI-inhomogeneities (Fig. 9e) indicate that the 1-D condition holds.

In Figs. 10a and b, we have plotted the UVI images for the two time steps of 23:41:58-23:42:35 UT and 23:45:0223:45:39 UT. Both images reveal more or less uniform conditions, with the latter changing only $10 \%$ in UVI intensity in the east-west direction within the STARE FOV. The two images also suggest uniform conditions throughout the entire region of $\sim 800 \mathrm{~km}$, as the situation is much the same within
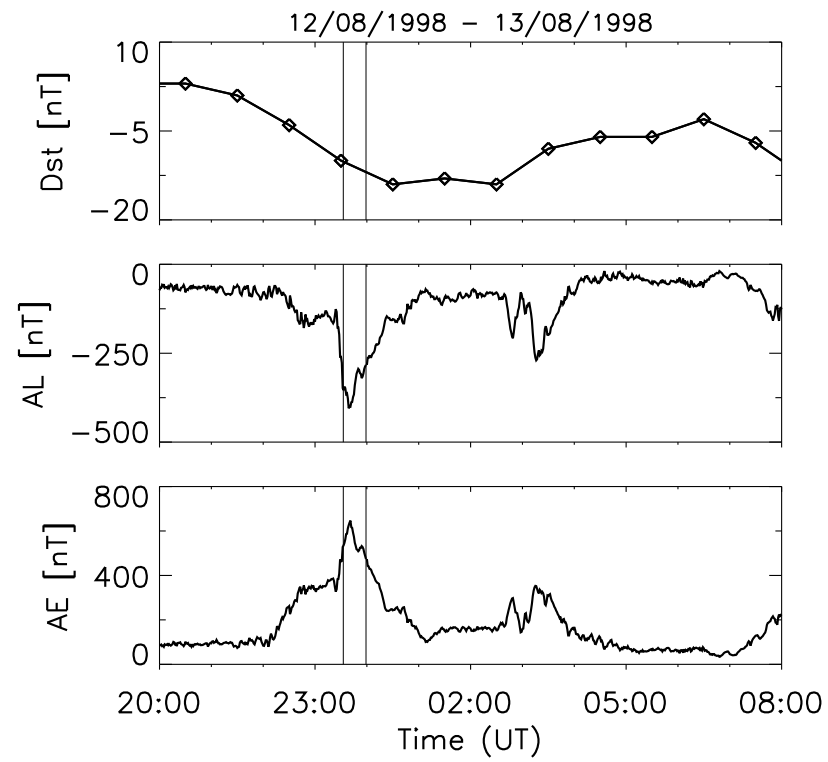

Fig. 8. The AE, AL and $D_{s t}$-indices between 20:00 and 08:00 UT on 12-13 August 1998. The two vertical solid lines indicate our time interval with data between 23:30 and 23:55 UT.

the outer black box corresponding to the PIXIE resolution. We therefore see in Fig. 9f that the differences in \% between the average UVI intensity for a region of $470 \mathrm{~km} \times 380 \mathrm{~km}$ (the STARE FOV) and $\sim 800 \mathrm{~km}$ (the PIXIE resolution) are practically 0 . The results show that all four time steps occur during relatively uniform ionospheric conditions, and that all the conductance sets provide differences of less than $\pm 30 \%$.

\section{Summary}

In this study we have derived the ionospheric Hall and Pedersen conductances during three periods with substorm activity in 1998 using two different techniques. One method involves remote sensing of UV and X-ray emissions from space, using data from the UVI and PIXIE cameras on board the Polar satellite. We first derive instantaneous global maps of the precipitating electron energy spectra from less than $1 \mathrm{keV}$ to $100 \mathrm{keV}$. We then derive height profiles of the resulting ionization and calculate the conductances. In another approach, we use data from the MIRACLE ground-based network in Northern Scandinavia. Mesoscale instantaneous conductance profiles can be inferred using a technique named the 1-D method of characteristics, which includes electric field measurements of the STARE coherent scatter radar and ground magnetometer data of the IMAGE network. While UVI/PIXIE measurements provide a single value of Hall and Pedersen conductances for the whole STARE FOV integrated over $\sim 4.5 \mathrm{~min}$, the 1-D method of characteristics establishes conductances every $20 \mathrm{~s}$ with a spatial resolution of $\sim 50 \mathrm{~km}$. To perform a comparison, conductances derived by the 1-D method of characteristics have been averaged in time over the 


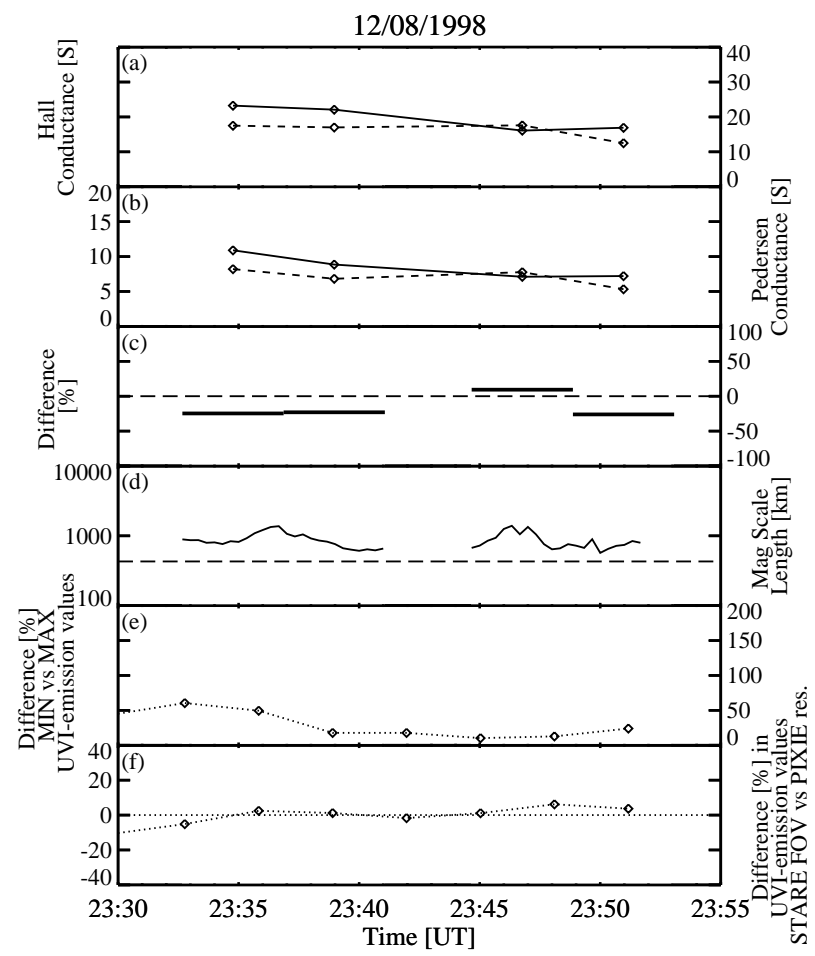

Fig. 9. (a) The Hall and (b) Pedersen conductances [S] derived in the Scandinavian region using UVI and PIXIE measurements (solid line) and the 1-D method of characteristics (dashed line) on $12 \mathrm{Au}-$ gust 1998. (c) The difference in \% between the two conductance sets. The horizontal dashed line is drawn to give the 0-percent level, while the length of the lines indicates the integration time period. (d) Magnetic field scale lengths $(\mathrm{km})$ between the two MIRACLE magnetometer stations of AND and KEV. (e) The average percentage calculated using the lowest and largest UVI-LBHL emission values at four strips of constant latitude within the STARE FOV. (f) The difference in $\%$ for the average UVI-LBHL intensity within the STARE FOV compared with the average UVI-LBHL intensity when including a larger area surrounding the STARE FOV matching the PIXIE resolution.

UVI/PIXIE 4.5-min intervals and in space over the STARE FOV.

The results presented in Sect. 3 reveal that the two techniques used to derive the conductances sometimes provide similar values. Other times, however, the conductances can differ strongly. For the events on 5 May and 26 June 1998, the best correspondence between the two conductance sets is found at the end of the investigated time periods, when we are in the late recovery phase of a substorm. Then the geomagnetic conditions are less disturbed.

An even better agreement is found for the conductances derived on 12 August 1998. As shown in Fig. 8, we start out in the substorm expansion phase, followed by the beginning of the recovery phase. The $\mathrm{AE}$ and $\mathrm{AL}$ indices, though, are moderate compared to the other two events. We further note that this substorm takes place during non-storm conditions, while the events on 5 May and 26 June 1998, occurred during the main phase of an intense geomagnetic storm. (a)

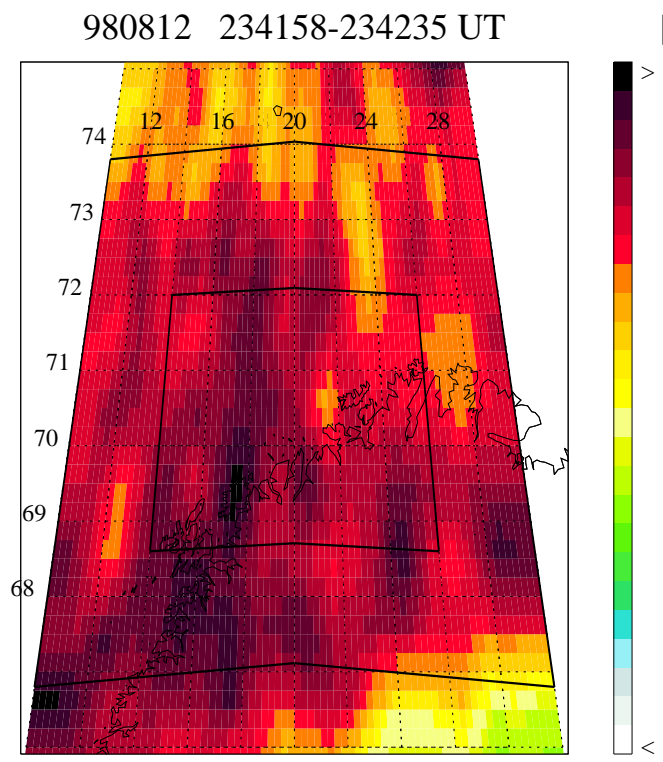

[R]

996

910

736

476

390

303

216

130

(b)

$980812 \quad 234502-234539$ UT

[R]

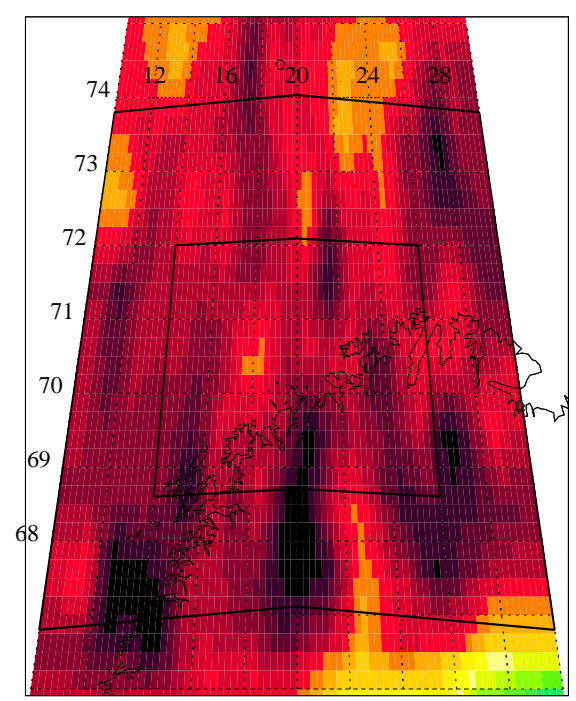

823

752

680

608

Fig. 10. The UVI-LBHL images for the two time steps of (a) 2341:58-2342:35 UT and (b) 23:45:02-23:45:39 UT on 12 August 1998. The inner black box drawn on top of the images corresponds to the STARE FOV, while the outer box matches the PIXIE resolution of $\sim 800 \mathrm{~km}$.

The largest deviations between the two conductance sets are found on 5 May and 26 June 1998, just after the maximum in $\mathrm{AE}$, meaning that highly disturbed conditions regarding particle precipitation, electric fields and currents are expected. Such ionospheric conditions are not favorable for the two procedures including the UVI/PIXIE technique and the 1-D method of characteristics. As explained in Sect. 2, the two techniques should provide reliable results during times with fairly uniform ionospheric conditions. A number of difficulties arise when too much ionospheric turbulence occurs. 
This has led to the separation of the different conductance values presented in Figs. 3, 6 and 9, according to their corresponding ionospheric conditions. Relatively uniform conditions have been attributed to situations when the following selection criteria are fulfilled: 1) More than $70 \%$ of the individual magnetic scale lengths (Figs. 3d, 6d, 9d) must be larger than $429 \mathrm{~km}$. 2) The largest UVI-inhomogeneity within the STARE FOV (Figs. 3e, 6e, 9e) must be less than $100 \%$. 3) The difference between the average UVI intensity (Figs. 3f, 6f, 9f) within the STARE FOV vs. a larger region of $\sim 800 \mathrm{~km}$ (the PIXIE resolution) must be less than $20 \%$.

For the event on 5 May we find that 6 time periods may be attributed to relatively uniform conditions. Five of these cases provide conductances with differences of less than $\pm 30 \%$. The same holds true for the 5 time steps with local turbulence below our tolerance limit during the event on 26 June, as well as all 4 values taken from 12 August. On the contrary, only 4 of the conductance pairs from the remaining 9 periods with non-uniform conditions reveal differences of less than $\pm 30 \%$.

In Fig. 11, we show the relation between the three selection criteria and the differences between the two conductance sets. The vertical axis gives the percentage of individual magnetic scale lengths larger than $429 \mathrm{~km}$, while the UVI-inhomogeneities are provided along the horizontal axis. We note that 15 of the values plotted have percentages larger than $70 \%$ (above the dotted horizontal line) and UVI-inhomogeneities less than $100 \%$ (left side of the dotted vertical line). These values are assigned relatively uniform conditions, and the red and dark red colors filling 14 of the 15 boxes reveal differences between the two conductance sets of less than $\pm 30 \%$. The 9 remaining data points marked by crosses take place during non-uniform conditions. In general, these values give significantly larger differences, as demonstrated by the orange, yellow and green colors. We also note that a thick horizontal line goes through three of these data points during non-uniform conditions. This means that they have failed to satisfy the third selection criterion regarding the difference between the average UVI intensity for the STARE FOV vs. a larger region of $\sim 800 \mathrm{~km}$ (the PIXIE resolution).

In Fig. 12, we present three scatter plots showing the correspondence between the two Hall conductance sets during the different ionospheric conditions. When including all 24 data points available we obtain a correlation coefficient of 0.27 (Fig. 12a). Then we plot the 15 cases of relatively uniform ionospheric conditions, resulting in a much higher correlation coefficient of 0.57 (Fig. 12b). The remaining 9 data points represents non-uniform conditions. These are plotted in Fig. 12c, and the correlation coefficient drops to 0.02 .

\section{Conclusion}

The ionospheric conductances have been derived and compared for three periods with substorm activity in 1998, using remote sensing of UV- and X-ray emissions (Sect. 2.1) and
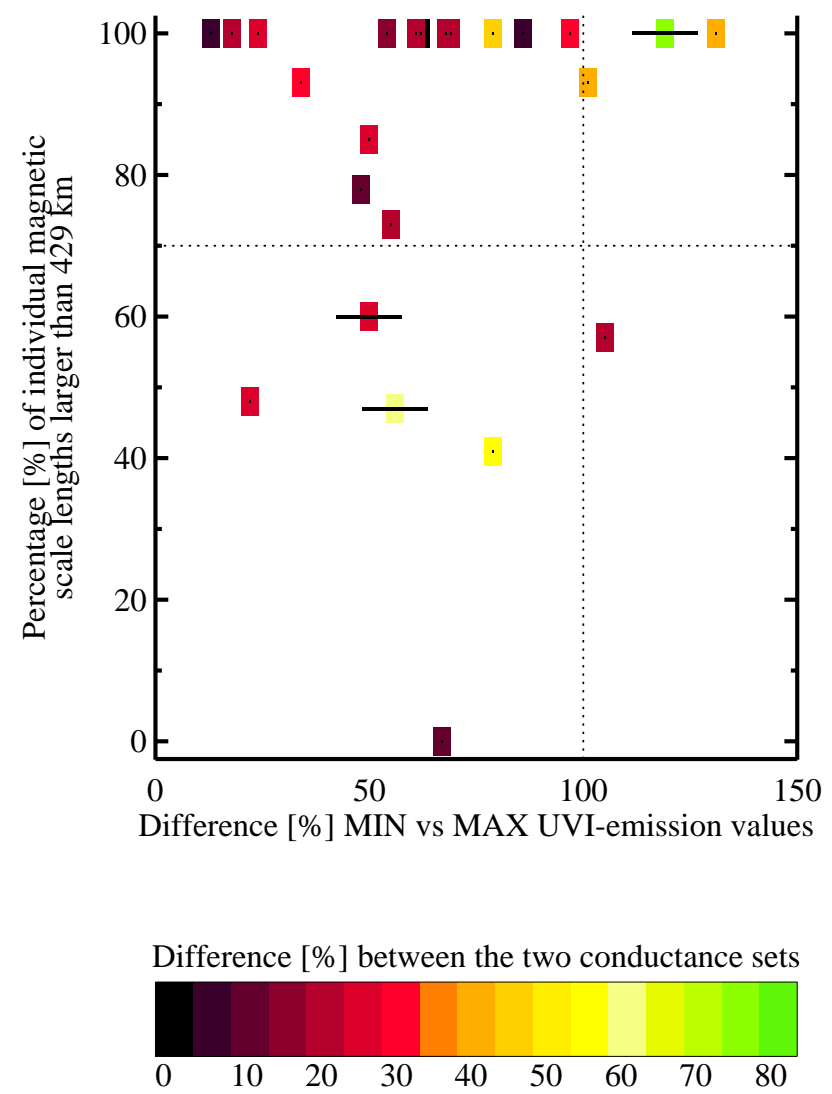

Fig. 11. The correspondence between percentage of individual magnetic scale lengths larger than $429 \mathrm{~km}$ (vertical axis), UVIinhomogeneities (horizontal axis), and the differences between the two conductance sets (color bar). The data points assigned nonuniform conditions are marked by crosses. To fulfill the first two selection criteria, the data points must be above the dotted horizontal line at $70 \%$ (selection criterion No. 1) and the left side of the dotted vertical line at $100 \%$ (selection criterion No. 2). The thick horizontal lines through three of the boxes indicate the data points which do not fulfill selection criterion No. 3 .

the 1-D method of characteristics (Sect. 2.2), respectively. In general, the conductances derived using the two methods do not correlate. However, when carefully identifying the data points during relatively uniform conditions, the results indicate that the two very different techniques provide fairly similar conductance values. By imposing constraints on how much local turbulence we will tolerate, we define relatively uniform and non-uniform ionospheric conditions. We find that 15 of the 24 cases fulfill our selection criteria. Fourteen of these 15 data points provide conductances with differences of less than $\pm 30 \%$. On the contrary, only 4 of the remaining 9 periods with non-uniform conditions reveal differences of less than $\pm 30 \%$. A correlation coefficient of 0.27 is found for the two Hall conductance sets when including all 24 individual data points analyzed. By constraining our data set to include only data when the local turbulence is below a certain level, the correlation coefficient rises to 0.57 . 


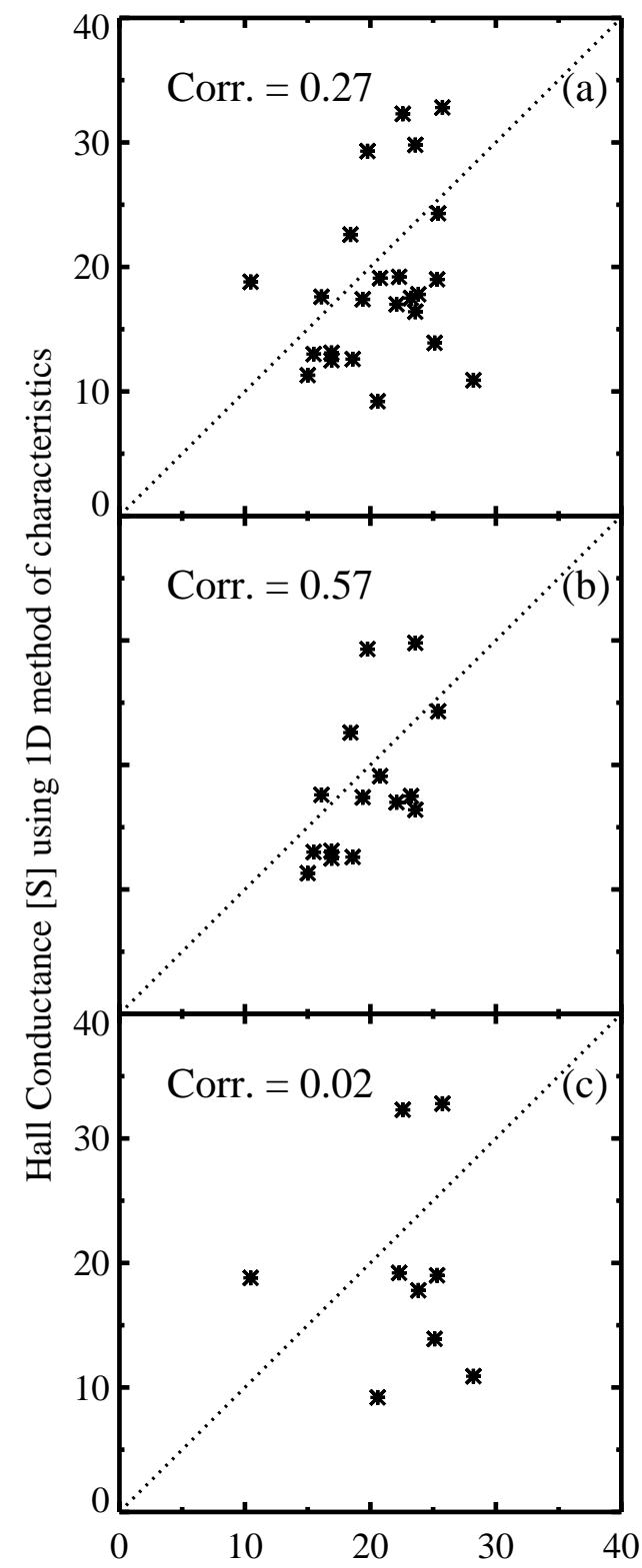

Hall Conductance [S] using the UVI/PIXIE technqiue

Fig. 12. Hall conductances $\Sigma_{H}$ derived using the UVI/PIXIE technique (horizontal axis) and the 1-D method of characteristics (vertical axis) during (a) all conditions (24 data points), (b) relatively uniform conditions (15 data points), and (c) non-uniform conditions (9 data points). The dotted line having a proportionality factor of 1.0 is drawn to indicate the locations of the Hall conductance values in cases where the two data sets provide the same output.

As discussed in Sect. 1, the ionospheric conductances can be estimated using many different techniques. The reliability of the methods used in this study, involving remote sensing of UV- and X-ray emissions (Sect. 2.1) and 1-D method of characteristics (Sect. 2.2), depends on several assumptions and limitations. Our database is further restricted to 24 data points, meaning that we must be cautious when interpreting the results. Despite the limited statistics we nevertheless find that the relation between the conductance sets is significantly improved when only including cases with relatively uniform conditions. As discussed in Sect. 2.1, studies by Germany et al. (2001) and Østgaard et al. (2001) indicate that the energy characteristics derived from UVI and PIXIE measurements, most important for the conductance calculations, are fairly reliable. This suggests that the estimated conductances using Polar satellite data provide a meaningful representation of the actual conductivities. The same conclusion can be made for the method of characteristics, according to investigations by Amm (1995) and Amm et al. (2003). The fairly good agreement seen in Fig. 12b supports the two very different techniques used and puts greater confidence in the two methods.

As explained in Sect. 1, it is not self-evident that simple averaging of the mesoscale results (1-D method of characteristics) allows for a continuous transition to the large-scale results (the UVI/PIXIE technique). By regarding the two sets of conductances as results of stochastical processes that operate on different scales, a number of different situations can be imagined where averaging would not work. The correlation coefficient between the two conductance sets of 0.57 found in this study during relatively uniform conditions is not impressive, but still indicates that a reasonable agreement between the two methods giving large-scale and mesoscale conductances can be reached. Therefore, it makes sense to study the ionospheric electrodynamics combining data with large spatial and temporal differences in the scales of the measurements. The lack of correspondence during non-uniform conditions also demonstrates the limitations of the two procedures, suggesting that the methods are less reliable when the local ionospheric region is in a highly turbulent state.

Acknowledgements. This study was supported by the Research Council of Norway (NFR). The work of O. A. was supported by the Academy of Finland. The work of G. A. Germany was supported by Subaward SA3527 from the University of California in Berkeley and by NASA AISRP Grant NAG5-1074. A. A. thanks A. D. Richmond for useful discussions on collision frequencies.

Topical Editor M. Lester thanks J. Watermann and another referee for their help in evaluating this paper.

\section{References}

Aksnes, A., Stadsnes, J., Bjordal, J., Østgaard, N., Vondrak, R. R., Detrick, D. L., Rosenberg, T. J., Germany, G. A., and Chenette, D.: Instantaneous ionospheric global conductance maps during an isolated substorm, Ann. Geophys., 20, 1181, 2002,

SRef-ID: 1432-0576/ag/2002-20-1181.

Aksnes, A., Stadsnes, J., Lu, G., Østgaard, N., Vondrak, R. R., Detrick, D. L., Rosenberg, T. J., Germany, G. A., and Schulz, M.: Effects of energetic electrons on the electrodynamics in the ionosphere, Ann. Geophys., 22, 475, 2004,

SRef-ID: 1432-0576/ag/2004-22-475.

Amm, O.: Direct determination of the local ionospheric Hall conductance distribution from two-dimensional electric and magnetic field data: application of the method using models of typical ionospheric electrodynamic situations, J. Geophys. Res., 100, 241731995. 
Amm, O.: Method of characteristics in spherical geometry applied to a Harang-discontinuity situation, Ann. Geophys., 16, 413, 1998 ,

\section{SRef-ID: 1432-0576/ag/1998-16-413.}

Amm, O., Aikio, A., Bosqued, J. -M., Dunlop, M., Fazakerley, A., Janhunen, P., Kauristie, K., Lester, M., Sillanpää, I., Taylor, M. G. G. T., Vontrat-Reberac, A., Mursula, K., and André, M.: Mesoscale structure of a morning sector ionospheric shear flow region determined by conjugate Cluster II and MIRACLE ground-based observations, Ann. Geophys., 21, 1737, 2003, SRef-ID: 1432-0576/ag/2003-21-1737.

Banks, P. M. and Doupnik, J. R.: A review of auroral zone electrodynamics deduced from incoherent scatter radar observations, J. Atmos. Terr. Phys., 37, 951, 1975.

Basu, B. and Jasperse, J. R.: Linear transport theory of auroral proton precipitation: A comparison with observations, J. Geophys. Res., 92, 5920, 1987.

Baumjohann, W., Pellinen, R. J., Opgenoorth, H. J., and Nielsen, E.: Joint two-dimensional observations of ground magnetic and ionospheric electric fields associated with auroral zone currents: current system associated with local auroral break-ups, Planet. Space Sci., 29, 431, 1981.

Bostrøm, R.: A model of the auroral electrojets, J. Geophys. Res., 69, 4983, 1964.

Brekke, A.: Physics of the Upper Polar Atmosphere, John Wiley and Sons Ltd., Chichester, England, 1997.

Brekke, A., Doupnik, J. R., and Banks, P. M.: Incoherent scatter measurements of $\mathrm{E}$ region conductivities and currents in the auroral zone, J. Geophys. Res., 79, 3773, 1974.

Brekke, A., Hall, C., and Hansen, T. L.: Auroral ionospheric conductances during disturbed conditions, Ann. Geophys., 7, 269, 1989.

Buchert, S., Baumjohann, W., Haerendel, G., La Hoz, C., and Lühr, H.: Magnetometer and incoherent scatter observations of an intense Ps 6 pulsation event, J. Atmos. Terr. Phys., 50, 357, 1988.

Christakos, G.: Random Field Models in Earth Sciences, Academic Press, San Diego, USA, 1992.

Davies, J. A. and Lester, M.: The relationship between electric fields, conductances and currents in the high-latitude ionosphere: a statistical study using EISCAT data, Ann. Geophys., 17, 43, 1999 ,

\section{SRef-ID: 1432-0576/ag/1999-17-43.}

de la Beaujardière, O., Vondrak, R., Heelis, R., Hanson, W., and Hoffman, R.: Auroral arc electrodynamic parameters measured by AE-C and the Chatanika radar, J. Geophys. Res., 86, 4671, 1981.

Doe, R. A., Kelly, J. D., Lummerzheim, D., Parks, G. K., Brittnacher, M. J., Germany, G. A., and Spann, J.: Initial comparison of POLAR UVI and Sondrestrom IS radar estimates for auroral electron energy flux, Geophys. Res. Lett., 24, 999, 1997.

Duschek, A. and Hochrainer, A.: Tensorrechnung in analytischer darstellung II: Tensoranalysis, Springer-Verlag, New York, 1961.

Dymond, K. F., Budzien, S. A., and Thonnard, S. E.: Electron densities determined by the HIRAAS experiment and comparisons with ionosonde measurements, Geophys. Res. Lett., 28, 927, 2001.

Fukushima, N.: Generalized theorem for no ground magnetic effect of vertical currents connected with Pedersen currents in the uniform-conductivity ionosphere, Rep. Ionos. Space Res., Japan, 30, 35, 1976.

Gagnepain, J., Crochet, M., and Richmond, A. D.: Comparisoon of equatiorial electrojet models, J. Atmos. Terr. Phys., 39, 1119,
1977.

Germany, G. A., Torr, M. R., Richards, P. G., and Torr, D. G.: The dependence of modeled OI 1356 and N2 LBH auroral emissions on the neutral atmosphere, J. Geophys. Res., 95, 7725, 1990.

Germany, G. A., Parks, G. K., Brittnacher, M., Cumnock, J., Lummerzheim, D., Spann, J. F., Chen, L., Richards, P. G., and Rich, F. J.: Remote determination of auroral energy characteristics during substorm activity, Geophys. Res. Lett., 24, 995, 1997.

Germany, G. A., Parks, G. K., Brittnacher, M., Spann, J. F., Cumnock, J., Lummerzheim, D., Rich, F. J., and Richards, P. G.: Energy characterization of a dynamic auroral event using GGS UVI images, in Geospace Mass and Energy Flow: Results from the International Solar-Terrestrial Physics Program, edited by J. L. Horwitz, D. L. Gallagher, and W. K. Peterson, 143, AGU, 104 Washington, D.C., 1998a.

Germany, G. A., Spann, J. F., Parks, G. K., Brittnacher, M., Elsen, R., Chen, L., Lummerzheim, D., and Rees, M.: Auroral observations from the Polar Ultraviolet Imager (UVI), in Geospace Mass and Energy Flow: Results from the International SolarTerrestrial Physics Program, edited by J. L. Horwitz, D. L. Gallagher, and W. K. Peterson, 149, AGU, 104, Washington, D.C., 1998b.

Germany, G. A., Lummerzheim, D., and Richards, P. G.: Impact of model differences on quantitative analysis of FUV auroral emissions: Total ionization cross sections, J. Geophys. Res., 106, $12837,2001$.

Gonzalez, W. D., Joselyn, J. A., Kamide, Y., Kroehl, H., Rostoker, G., Tsurutani, B. T., and Vasyliunas, V. M.: What is a geomagnetic storm?, J. Geophys. Res., 99, 5771, 1994.

Imhof, W. L., Spear, K. A., Hamilton, J. W., Higgins, B. R., Murphy, M. J., Pronko, J. G., Vondrak, R. R., McKenzie, D. L., Rice, C. J., Gorney, D. J., Roux, D. A., Williams, R. L., Stein, J. A., Bjordal, J., Stadsnes, J., Njøten, K., Rosenberg, T. J., Lutz, L., and Detrick, D. L.: The Polar Ionospheric X-ray Imaging Experiment (PIXIE), Space Sci. Rev., 71, 385, 1995.

Inhester, B., Untiedt, J., Segatz, M., and Kürschner, M.: Direct determination of the local ionospheric Hall conductance distribution from two-dimensional electric and magnetic field data, J. Geophys. Res., 97, 4073, 1992.

Itikawa, Y.: Effective collision frequency of electrons in atmospheric gases, Planet. Space Sci., 19, 993, 1971.

Kamide, Y. and Vickrey, J. F.: Relative contribution of ionospheric conductivity and electric field to the auroral electrojets, J. Geophys. Res., 88, 7989, 1983.

Kamide, Y., Craven, J. D., Frank, L. A., Ahn, B. -H., and Akasofu, S. -I.: Modeling substorm current systems using conductivity distributions inferred from DE auroral images, J. Geophys. Res., 91, 112351986.

Krall, N. A. and Trivelpiece, A. W.: Principles of Plasma Physics, McGraw-Hill, New York, USA, 1973.

Lorence, L. J., CEPXS/ONELD Version 2.0: A discrete ordinates code package for general one-dimensional coupled electronphoton transport, IEES Trans. Nucl. Sci, 39, 1031, 1992.

Lühr, H., Aylward, A., Bucher, S. C., Pajunpää, A., Pajunpää, K., Holmboe, T., and Zalewski, S. M.: Westward moving dynamic substorm features observed with the IMAGE magnetometer network and other ground-based instruments, Ann. Geophys., 16, 425, 1998,

SRef-ID: 1432-0576/ag/1998-16-425.

Lummerzheim, D., Rees, M. H., Craven, J. D., and Frank, L. A.: Ionosheric conductances derived from DE-1 auroral images, J. Atmos. Terr. Phys., 53, 281, 1991. 
Marklund, G., Sandahl, I., and Opgenoorth, H.: A study of the dynamics of a discrete auroral arc, Planet. Space Sci., 30, 179, 1982.

Mason, E. A.: Estimated ion mobilities for some air constituents, Planet. Space Sci., 18, 137, 1970.

Mersmann, U., Baumjohann, W., Küppers, F., and Lange, K.: Analysis of an eastward electrojet by means of upward continuation of ground-based magnetometer data, J. Geophys., 45, 281, 1979.

Olsson, A., Persson, M. A. L., Opgenoorth, H. J., and Kirkwood, S.: Particle precipitation in auroral breakups and westward traveling surges, J. Geophys. Res., 101, 24 661, 1996.

Opgenoorth, H. J., Pellinen, R. J., Baumjohann, W., Nielsen, E., Marklund, G., and Eliasson, L.: Three-dimensional current flow and particle precipitation in a westward travelling surge (observed during the barium-GEOS rocket experiment), J. Geophys. Sci., 88, 3138, 1983.

Østgaard, N., Stadsnes, J., Bjordal, J., Vondrak, R. R., Cummer, S. A., Chenette, D., Schulz, M., and Pronko, J.: Cause of the localized maximum of X-ray emission in the morning sector: A comparison with electron measurements, J. Geophys. Res., 105, $20869,2000$.

Østgaard, N., Stadsnes, J., Bjordal, J., Germany, G. A., Vondrak, R. R., Parks, G. K., Cummer, S. A., Chenette, D., and Pronko, J.: Auroral electron distributions derived from combined UV and X-ray emissions, J. Geophys. Res., 106, 26 081, 2001.

Østgaard, N., Germany, G. A., Stadsnes, J., and Vondrak, R. R.: Energy analysis of substorms based on remote sensing techniques, solar wind measurements and geomagnetic indices, J. Geophys. Res., 107, 1233, 2002.

Pesnell, W. D., Omidvar, K., and Hoegy, W. R.: Momentum transfer collision frequency of O+-O, Geophys. Res. Lett., 20, 1343, 1993.

Pulkkinen, A., Amm, O., and Viljanen, A.: and BEAR working group, Ionospheric equivalent current distributions determined with the method of spherical elementary current systems, J. Geophys. Res., 108, 1053, doi:10.1029/2001JA005085, 2003.

Rees, M. H.: Auroral ionization and excitation by incident energetic electrons, Planet. Space. Sci., 11, 1209, 1963.

Rees, M. H., Lummerzheim, D., Roble, R. G., Winningham, J. D., Craven, J. D., and Frank, L. A.: Auroral energy deposition rate, characteristic electron energy, and ionospheric parameters derived from Dynamics Explorer 1 images, J. Geophys. Res., 93, $12841,1988$.

Robinson, R. M. and Vondrak, R. R.: Measurements of E region ionization and conductivity produced by solar illumination at high latitudes, J. Geophys. Res., 89, 3951, 1984.
Robinson, R. M. and Vondrak, R. R., Validation of techniques for space based remote sensing of auroral precipitation and its ionospheric effects, Space Sci. Rev., 69, 331, 1994.

Robinson, R. M., Vondrak, R. R., Miller, K., Dabbs, T., and Hardy, D.: On calculating ionospheric conductances from the flux and energy of precipitating electrons, J. Geophys. Res., 92, 2565, 1987.

Schlegel, K.: Auroral zone E-region conductivities during solar minimum derived from EISCAT data, Ann. Geophys., 6, 129, 1988.

Senior, C.: Solar and particle contributions to auroral heightintegrated conductivities from EISCAT data: a statistical study, Ann. Geophys., 9, 449, 1991.

Sillanpää, I.: One-dimensional method of characteristics to determine ionospheric conductances and currents, unpublished Graduate Thesis, Helsinki, Finland, 2002.

Torr, M. R., Torr, D. G., Zukic, M., Johnson, R. B., Ajello, J., Banks, P., Clark, K., Cole, K., Keffer, C., Parks, G., Tsurutani, B., and Spann, J.: A far ultaviolet imager for the international solar-terrestrial physics mission, Space Sci. Rev., 71, 329, 1995.

Untiedt, J. and Baumjohann, W.: Studies of polar current systems using the IMS scandinavian magnetometer array, Space Sci. Rev., 63, 245, 1993.

Vasyliunas, V. M.: Mathematical models of magnetospheric convection and its coupling to the ionosphere, in Particles and Fields in the Magnetosphere, edited by B. M. McCormac, 60, D. Reidel, Hinghamn, Mass., 1970.

Viehland, L. A. and Mason, E. A.: Transport Properties of Gaseous Ions Over a Wide Energy Range - Part IV, Atomic Data and Nuclear Data Tables, 60, 37, 1995.

Vondrak, R. and Robinson, R., Inference of high-latitude ionization and conductivity from AE-C measurements of auroral electron fluxes, J. Geophys. Res., 90, 7505, 1985.

Vondrak, R. R. and Baron, M. J.: Radar measurements of the latitudinal variation of auroral ionization, Radio Sci., 11, 939, 1976.

Vondrak, R. R., Robinson, R. M., Mizera, P. F., and Gorney, D. J.: X-ray spectrophotometric remote sensing of diffuse auroral ionization, Radio Sci., 23, 537, 1988.

Watermann, J., de la Beaujardière, O., and Rich, F. J.: Comparison of ionospheric electrical conductances inferred from coincident radar and spacecraft measurements and photoionization models, J. Atmos. Terres. Phys., 55, 1513, 1993.

Wedde, T., Doupnik, J. R., and Banks, P. M.: Chatanika observations of the latitudinal structure of electric fields and particle precipitation on 21 November 1975, J. Geophys. Res., 82, 2743, 1977. 